\title{
Deriving Filtering Algorithms from Constraint Checkers
}

\author{
Nicolas Beldiceanu ${ }^{1}$, Mats Carlsson ${ }^{2}$, and Thierry Petit ${ }^{1}$ \\ 1 LINA FRE CNRS 2729, École des Mines de Nantes, FR-44307 Nantes Cedex 3, France. \\ \{Nicolas.Beldiceanu, Thierry.Petit\}@emn.fr \\ 2 SICS, P.O. Box 1263, SE-164 29 Kista, Sweden. \\ Mats.Carlssonesics.se
}

April 27, 2004

SICS Technical Report T2004:08

ISRN: SICS-T-2004/08-SE

ISSN: $1100-3154$

\begin{abstract}
This reportdeals with global constraints for which the set of solutions can be recognized by an extended finite automaton whose size is bounded by a polynomial in $n$, where $n$ is the number of variables of the corresponding global constraint. By reformulating the automaton as a conjunction of signature and transition constraints we show how to systematically obtain a filtering algorithm. Under some restrictions on the signature and transition constraints this filtering algorithm achieves arc-consistency. An implementation based on some constraints as well as on the metaprogramming facilities of SICStus Prolog is available. For a restricted class of automata we provide a filtering algorithm for the relaxed case, where the violation cost is the minimum number of variables to unassign in order to get back to a solution. Keywords: Constraint Programming, Global Constraints, Filtering Algorithms, Finite Automata, Relaxation.
\end{abstract}

\section{Introduction}

Deriving filtering algorithms for global constraints is usually far from obvious and requires a lot of energy. As a first step toward a methodology for semi-automatic development of filtering algorithms for global constraints, Carlsson and Beldiceanu have introduced [12] an approach to design filtering algorithms by derivation from a finite automaton. As quoted in their discussion, constructing the automaton was far from obvious since it was mainly done as a rational reconstruction of an emerging understanding of the necessary case analysis related to the required pruning. However, it is commonly admitted that coming up with a checker which tests whether a ground instance is a solution or not is usually straightforward. This was for instance done for constraints defined in extension first by Vempaty [29] and later on by Amilhastre et al. [2]. This was also done for arithmetic constraints by Boigelot and Wolper [10]. Within the context of global constraints on a finite sequence of variables, the recent work of Pesant [26] uses also a finite automaton for constructing a filtering algorithm. This reportfocuses on 
those global constraints that can be checked by scanning once through their variables without using any extra data structure.

As a second step toward a methodology for semi-automatic development of filtering algorithms, we introduce a new approach which only requires defining a finite automaton that checks a ground instance. We extend traditional finite automata in order not to be limited only to regular expressions. Our first contribution is to show how to reformulate the automaton associated with a global constraint as a conjunction of signature and transition constraints. We characterize some restrictions on the signature and transition constraints under which the filtering algorithm induced by this reformulation achieves arc-consistency and apply this new methodology to the three following problems:

- The design of filtering algorithms for a fairly large set of global constraints.

- The design of filtering algorithms for handling the conjunction of several global constraints.

- The design of constraints for dealing with problems involving finite state systems where the transition diagram is known.

Our second contribution is to provide for a restricted class of automata a filtering algorithm for the relaxed case. This technique relies on the variable based violation cost introduced in $[27,24]$. This cost was advocated as a generic way for expressing the violation of a global constraint. However, algorithms were only provided for the soft_alldifferent constraint [27]. We come up with an algorithm for computing a sharp bound of the minimum violation cost and with a filtering algorithm for pruning in order to avoid to exceed a given maximum violation cost.

The rest of this reportis organized as follows. Section 2 describes the kind of finite automaton used for recognizing the set of solutions associated with a global constraint. Section 3 shows how to come up with a filtering algorithm which exploits the previously introduced automaton. Section 4 describes typical applications of this technique. For a restricted class of automata, Section 5 provides a filtering algorithm for the relaxed case. Finally, the Appendix gives the different automata.

\section{Description of the Automaton Used for Checking Ground Instances}

We first discuss the main issues behind the task of selecting what kind of automaton to consider for expressing in a concise way the set of solutions associated with a global constraint. We consider global constraints for which any ground instance can be checked in linear time by scanning once through their variables without using any data structure. In order to concretely illustrate this point we first select a set of global constraints and write down a checker for each of them. Finally, we give for each checker a sketch of the corresponding automaton. Based on these observations, we define the type of automaton we will use.

Selecting an Appropriate Description. As we previously said, we focus on those global constraints that can be checked by scanning once through their variables. This is for instance the case of element [20], minimum [4], pattern [11], global_con- 
tiguity [23], lexicographic ordering [18], among [6] and inflexion [3]. Since they illustrate key points needed for characterizing the set of solutions associated with a global constraint, our discussion will be based on the last four constraints for which we now recall the definition:

- The global_contiguity(vars) constraint enforces for the sequence of 0-1 variables vars to have at most one group of consecutive 1 . For instance, the constraint global_contiguity $([0,1,1,0])$ holds since we have only one group of consecutive 1.

- The lexicographic ordering constraint $\vec{x} \leq_{\text {lex }} \vec{y}$ over two vectors of variables $\vec{x}=$ $\left\langle x_{0}, \ldots, x_{n-1}\right\rangle$ and $\vec{y}=\left\langle y_{0}, \ldots, y_{n-1}\right\rangle$ holds iff $n=0$ or $x_{0}<y_{0}$ or $x_{0}=y_{0}$ and $\left\langle x_{1}, \ldots, x_{n-1}\right\rangle \leq_{\text {lex }}\left\langle y_{1}, \ldots, y_{n-1}\right\rangle$.

- The among(nvar, vars, values) constraint restricts the number of variables of the sequence of variables vars, which take their value in a given set values, to be equal to the variable nvar. For instance, among $(3,[4,5,5,4,1],[1,5,8])$ holds since exactly 3 values of the sequence 45541 are located in $\{1,5,8\}$.

- The inflexion(ninf, vars) constraint enforces the number of inflexions of the sequence of variables vars to be equal to the variable ninf. An inflexion is described by one of the following patterns: a strict increase followed by a strict decrease or, conversely, a strict decrease followed by a strict increase. For instance, inflexion $(4,[3,3,1,4,5,5,6,5,5,6,3])$ holds since we can extract from the sequence 33145565563 the four subsequences $314,565,6556$ and 563 , which all follow one of these two patterns.

Parts (A1), (B1), (C1) and (D1) of Fig. 1 depict the four checkers respectively associated with global_contiguity, with $\leq_{\text {lex }}$, with among and with inflexion. For each checker we observe the following facts:

- Within the checker depicted by part (A1) of Fig. 1, the values of the sequence $\operatorname{vars}[0], \ldots, \operatorname{vars}[n-1]$ are successively compared against 0 and 1 in order to check that we have at most one group of consecutive 1 . This can be translated to the automaton depicted by part (A2) of Fig. 1. The automaton takes as input the sequence $\operatorname{vars}[0], \ldots, \operatorname{vars}[n-1]$, and triggers successively a transition for each term of this sequence. Transitions labeled by 0,1 and $\$$ are respectively associated with the conditions vars $[i]=0$, vars $[i]=1$ and $i=n$. Transitions leading to failure are systematically skipped. This is why no transition labeled with a 1 starts from state $z$.

- Within the checker given by part (B1) of Fig. 1, the components of vectors $\vec{x}$ and $\vec{y}$ are scanned in parallel. We first skip all the components that are equal and then perform a final check. This is represented by the automaton depicted by part (B2) of Fig. 1. The automaton takes as input the sequence $\langle x[0], y[0]\rangle, \ldots,\langle x[n-$ 1], $y[n-1]\rangle$ and triggers a transition for each term of this sequence. Unlike the global_contiguity constraint, some transitions now correspond to a condition (e.g. $x[i]=y[i], x[i]<y[i]$ ) between two variables of the $\leq_{\text {lex }}$ constraint.

- Observe that the among(nvar, vars, values) constraint involves a variable nvar whose value is computed from a given collection of variables vars. The checker depicted by part (C1) of Fig. 1 counts the number of variables of vars $[0], \ldots, \operatorname{vars}[n-$ 


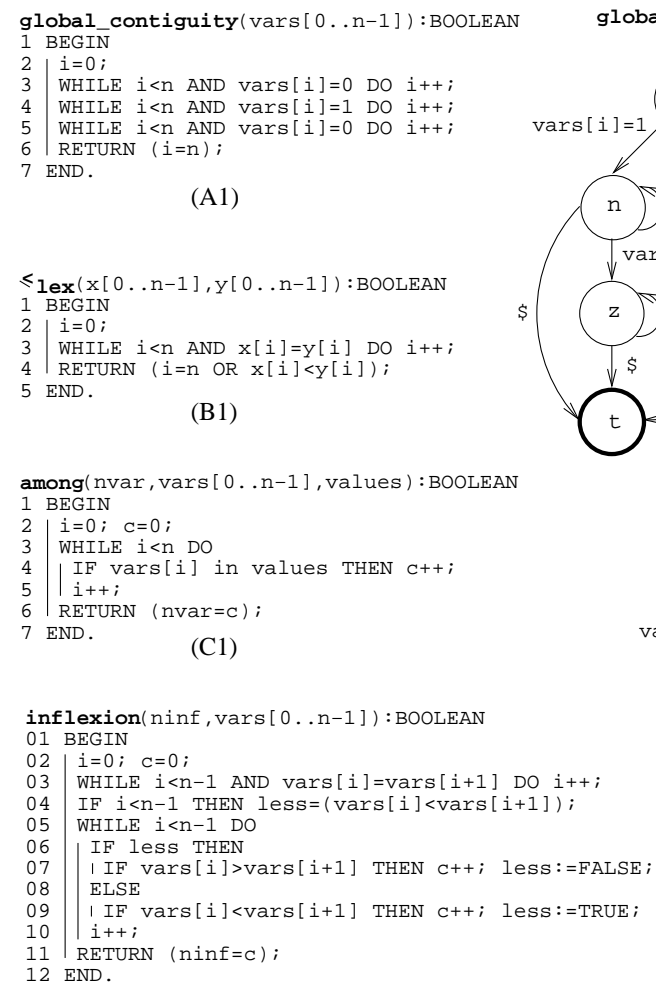

(D1)

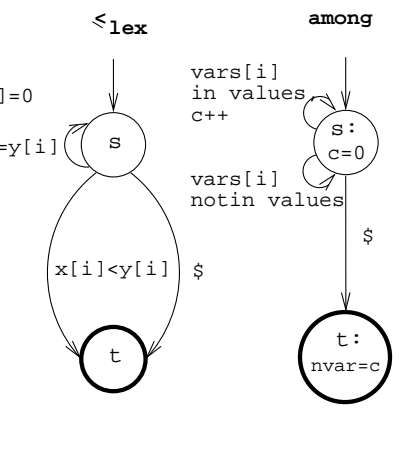

(B2)

(C2)

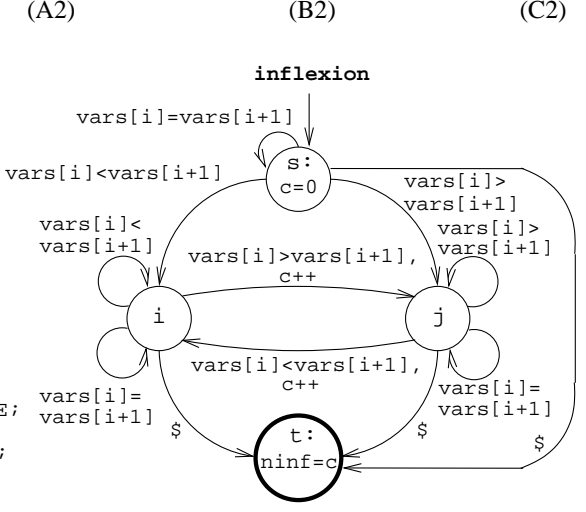

(D2)

Fig. 1. Four checkers and their corresponding automata. 
1] that take their value in values. For this purpose it uses a counter $c$, which is eventually tested against the value of nvar. This convinced us to allow the use of counters in an automaton. Each counter has an initial value which can be updated while triggering certain transitions. The final state of an automaton can enforce a variable of the constraint to be equal to a given counter. Part (C2) of Fig. 1 describes the automaton corresponding to the code given in part $(\mathrm{C} 1)$ of the same figure. The automaton uses the counter variable $c$ initially set to 0 and takes as input the sequence $\operatorname{vars}[0], \ldots, \operatorname{vars}[n-1]$. It triggers a transition for each variable of this sequence and increments $c$ when the corresponding variable takes its value in values. The final state returns a success when the value of $c$ is equal to nvar. At this point we want to stress the following fact: It would have been possible to use an automaton which avoids the use of counters. However, this automaton would depend on the effective value of the parameter nvar. In addition, it would require more states than the automaton of part (C2) of Fig. 1. This is typically a problem if we want to have a fixed number of states in order to save memory as well as time.

- As the among constraint, the inflexion(ninf, vars) constraint involves a variable ninf whose value is computed from a given sequence of variables $\operatorname{vars}[0], \ldots, \operatorname{vars}[n-1]$. Therefore, the checker depicted in part (D1) of Fig. 1 uses also a counter $c$ for counting the number of inflexions, and compares its final value to the ninf parameter. This program is represented by the automaton depicted by part (D2) of Fig. 1. It takes as input the sequence of pairs $\langle\operatorname{vars}[0], \operatorname{vars}[1]\rangle$, $\langle$ vars [1], vars [2] $\rangle, \ldots,\langle$ vars $[n-2]$, vars $[n-1]\rangle$ and triggers a transition for each pair. Observe that a given variable may occur in more than one pair. Each transition compares the respective values of two consecutive variables of vars [0..n-1] and increments the counter $c$ when a new inflexion is detected. The final state returns a success when the value of $c$ is equal to ninf.

Synthesizing all the observations we got from these examples leads to the following remarks and definitions for a given global constraint $\mathcal{C}$ :

- For a given state, no transition can be triggered indicates that the constraint $\mathcal{C}$ does not hold.

- Since all transitions starting from a given state are mutually incompatible all automata are deterministic. Let $\mathcal{M}$ denote the set of mutually incompatible conditions associated with the different transitions of an automaton.

- Let $\mathcal{S}_{0}, \ldots, \mathcal{S}_{m-1}$ denote the sequence of subsets of variables of $\mathcal{C}$ on which the transitions are successively triggered. All these subsets contain the same number of elements and refer to some variables of $\mathcal{C}$. Since these subsets typically depend on the constraint, we leave the computation of $\mathcal{S}_{0}, \ldots, \mathcal{S}_{m-1}$ outside the automaton. To each subset $\mathcal{S}_{i}$ of this sequence corresponds a variable $S_{i}$ with an initial domain ranging over $[\min , \min +|\mathcal{M}|-1]$, where $\min$ is a fixed integer. To each integer of this range corresponds one of the mutually incompatible conditions of $\mathcal{M}$. The sequences $S_{0}, \ldots, S_{m-1}$ and $\mathcal{S}_{0}, \ldots, \mathcal{S}_{m-1}$ are respectively called the signature and the signature argument of the constraint. The constraint between $S_{i}$ and the variables of $\mathcal{S}_{i}$ is called the signature constraint and is denoted by $\Psi_{\mathcal{C}}\left(S_{i}, \mathcal{S}_{i}\right)$.

- From a pragmatic point the view, the task of writing a constraint checker is naturally done by writing down an imperative program where local variables, assignment 
statements and control structures are used. This suggested us to consider deterministic finite automata augmented with local variables and assignment statements on these variables. Regarding control structures, we did not introduce any extra feature since the deterministic choice of which transition to trigger next seemed to be good enough.

- Many global constraints involve a variable whose value is computed from a given collection of variables. This convinced us to allow the final state of an automaton to optionally return a result. In practice, this result corresponds to the value of a local variable of the automaton in the final state.

Defining an Automaton. An automaton $\mathcal{A}$ of a constraint $\mathcal{C}$ is defined by a septuple

$\langle\mathcal{S}$ ignature, Signature Domain, $\mathcal{S}$ ignature $\mathcal{A}$ rg, Signature $\mathcal{A}$ rg $\mathcal{P}$ attern, $\mathcal{C}$ ounters, $\mathcal{S}$ tates, $\mathcal{T}$ ransitions $\rangle$

where:

- Signature is the sequence of variables $S_{0}, \ldots, S_{m-1}$ corresponding to the signature of the constraint $\mathcal{C}$.

- Signature Domain is an interval which defines the range of possible values of the variables of $\mathcal{S}$ ignature.

- Signature $\mathcal{A r g}$ is the signature argument $\mathcal{S}_{0}, \ldots, \mathcal{S}_{m-1}$ of the constraint $\mathcal{C}$. The link between the variables of $\mathcal{S}_{i}$ and the variable $S_{i}(0 \leq i<m)$ is done by writing down the signature constraint $\Psi_{\mathcal{C}}\left(S_{i}, \mathcal{S}_{i}\right)$ in such a way that arc-consistency is achieved. In our context this is done by using standard features of the CLP(FD) solver of SICStus Prolog [13] such as arithmetic constraints between two variables, propositional combinators or the global constraints programming interface.

- When used, Signature $\mathcal{A}$ rg $\mathcal{P}$ attern defines a symbolic name for each term of $\mathcal{S}$ ignature $\mathcal{A}$ rg. These names can be used within the description of a transition for expressing an additional condition for triggering the corresponding transition.

- Counters is the, possibly empty, list of all counters used in the automaton $\mathcal{A}$. Each counter is described by a term t(Counter, InitialValue, FinalVariable) where Counter is a symbolic name representing the counter, InitialValue is an integer giving the value of the counter in the initial state of $\mathcal{A}$, and FinalVariable gives the variable that should be unified with the value of the counter in the final state of $\mathcal{A}$.

- States is the list of states of $\mathcal{A}$, where each state has the form source $(i d), \operatorname{sink}(i d)$ or node $(i d) . i d$ is a unique identifier associated with each state. Finally, source $(i d)$ and $\operatorname{sink}(i d)$ respectively denote the initial and the final state of $\mathcal{A}$.

- $\mathcal{T}$ ransitions is the list of transitions of $\mathcal{A}$. Each transition $t$ has the form $\operatorname{arc}\left(i d_{1}\right.$, label, $\left.i d_{2}\right)$ or $\operatorname{arc}\left(i d_{1}\right.$, label, $i d_{2}$, counters $) . i d_{1}$ and $i d_{2}$ respectively correspond to the state just before and just after $t$, while label depicts the value that the signature variable should have in order to trigger $t$. When used, counters gives for each counter of $\mathcal{C}$ ounters its value after firing the corresponding transition. This value is specified by an arithmetic expression involving counters, constants, as well as usual arithmetic functions such as,+- , min or max. The order used in the counters list is identical to the order used in $\mathcal{C}$ ounters. 
Example 1. As an illustrative example we give the description of the automaton associated with the inflexion(ninf, vars) constraint. We have:

- Signature $=S_{0}, S_{1}, \ldots, S_{n-2}$,

- Signature Domain $=0 . .2$,

- Signature $\mathcal{A r g}=\langle\operatorname{vars}[0], \operatorname{vars}[1]\rangle, \ldots,\langle\operatorname{vars}[n-2], \operatorname{vars}[n-1]\rangle$,

- Signature $\mathcal{A} r g \mathcal{P}$ attern is not used,

- Counters $=\mathrm{t}(c, 0$, ninf $)$,

- States $=[\operatorname{source}(s), \operatorname{node}(i), \operatorname{node}(j), \operatorname{sink}(t)]$,

- Transitions $=[\operatorname{arc}(s, 1, s), \operatorname{arc}(s, 2, i), \operatorname{arc}(s, 0, j), \operatorname{arc}(s, \$, t), \operatorname{arc}(i, 1, i), \operatorname{arc}(i, 2, i)$, $\operatorname{arc}(i, 0, j,[c+1]), \operatorname{arc}(i, \$, t), \operatorname{arc}(j, 1, j), \operatorname{arc}(j, 0, j), \operatorname{arc}(j, 2, i,[c+1]), \operatorname{arc}(j, \$, t)]$.

The signature constraint relating each pair of variables $\langle\operatorname{vars}[i]$, vars $[i+1]\rangle$ to the signature variable $S_{i}$ is defined as follows: $\Psi_{\text {inflexion }}\left(S_{i}, \operatorname{vars}[i], \operatorname{vars}[i+1]\right) \equiv \operatorname{vars}[i]>\operatorname{vars}[i+1] \Leftrightarrow$ $S_{i}=0 \wedge \operatorname{vars}[i]=\operatorname{vars}[i+1] \Leftrightarrow S_{i}=1 \wedge \operatorname{vars}[i]<\operatorname{vars}[i+1] \Leftrightarrow S_{i}=2$. The sequence of transitions triggered on the ground instance inflexion $(4,[3,3,1,4,5,5,6,5,5,6,3])$ is $\frac{s}{c=0} \stackrel{3=3 \Leftrightarrow S_{0}=1}{\longrightarrow} s \stackrel{3>1 \Leftrightarrow S_{1}=0}{\longrightarrow} j \stackrel{1<4 \Leftrightarrow S_{2}=2}{c=1} i \stackrel{4<5 \Leftrightarrow S_{3}=2}{\longrightarrow} i \stackrel{5=5 \Leftrightarrow S_{4}=1}{\longrightarrow} i \stackrel{5<6 \Leftrightarrow S_{5}=2}{\longrightarrow}$ $i \stackrel{6>5 \Leftrightarrow S_{6}=0}{\longrightarrow} j \stackrel{5=5 \Leftrightarrow S_{7}=1}{\longrightarrow} j \stackrel{5<6 \Leftrightarrow S_{8}=2}{\longrightarrow} i \frac{6>3 \Leftrightarrow S_{9}=0}{c=4} j \stackrel{\$}{\rightarrow} \frac{t}{n i n f=4}$. Each transition gives the corresponding condition and, eventually, the value of the counter $c$ just after firing that transition.

\section{Filtering Algorithm}

The filtering algorithm is based on the following idea. For a given global constraint $\mathcal{C}$, one can think of its automaton as a procedure that repeatedly maps a current state $Q^{i}$ and counter vector $\vec{K}^{i}$, given a signature variable $S_{i}$, to a new state $Q^{i+1}$ and counter vector $\vec{K}^{i+1}$, until a terminal state is reached. We then convert this procedure into a transition constraint $\Phi_{\mathcal{C}}\left(Q^{i}, \vec{K}^{i}, S_{i}, Q^{i+1}, \vec{K}^{i+1}\right)$ as follows. Assuming that the automaton associated with $\mathcal{C}$ has $n a \operatorname{arcs} \operatorname{arc}\left(q_{1}, s_{1}, q_{1}^{\prime}, \overrightarrow{f_{1}}(\vec{K})\right), \ldots, \operatorname{arc}\left(q_{n a}, s_{n a}, q_{n a}^{\prime}, \overrightarrow{f_{n a}}(\vec{K})\right)$, the transition constraint has the following form, implemented with arithmetic, case ${ }^{1}$, and element constraints [13]:

$$
\bigvee\left\{\begin{array}{l}
\left(Q^{i}=q_{1}\right) \wedge\left(S_{i}=s_{1}\right) \wedge\left(Q^{i+1}=q_{1}^{\prime}\right) \wedge\left(\vec{K}^{i+1}=\vec{f}_{1}\left(\vec{K}^{i}\right)\right) \\
\vdots \\
\left(Q^{i}=q_{n a}\right) \wedge\left(S_{i}=s_{n a}\right) \wedge\left(Q^{i+1}=q_{n a}^{\prime}\right) \wedge\left(\vec{K}^{i+1}=\vec{f}_{n a}\left(\vec{K}^{i}\right)\right)
\end{array}\right.
$$

We can then arrive at a filtering algorithm for $\mathcal{C}$ by decomposing it into a conjunction of $\Phi_{\mathcal{C}}$ constraints, "threading" the state and counter variables through the conjunction. In addition to this, we need the signature constraints $\Psi_{\mathcal{C}}\left(S_{i}, \mathcal{S}_{i}\right)(0 \leq i<m)$ that relate each signature variables $S_{i}$ to the variables of its corresponding signature argument $\mathcal{S}_{i}$. Filtering for the constraint $\mathcal{C}$ is provided by the conjunction of all signature and transitions constraints, (s being the start state and $t$ being the end state):

\footnotetext{
${ }^{1}$ When no counter variable is used we only need a single case constraint to encode the disjunction expressed by the transition constraint. Since the case constraint [13, page 463] achieves arc-consistency, it follows that, in this context, the transition constraint achieves arcconsistency.
} 


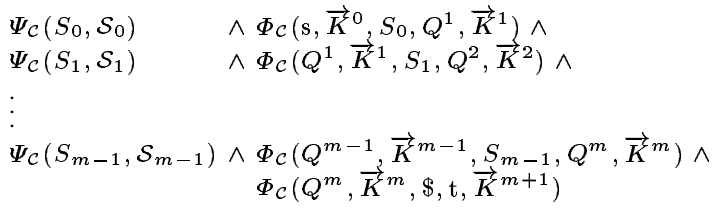

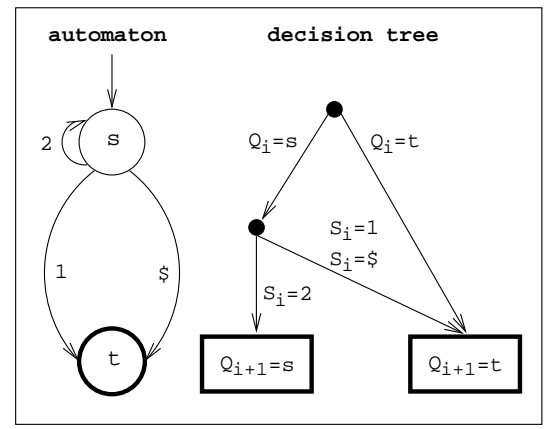

(A)

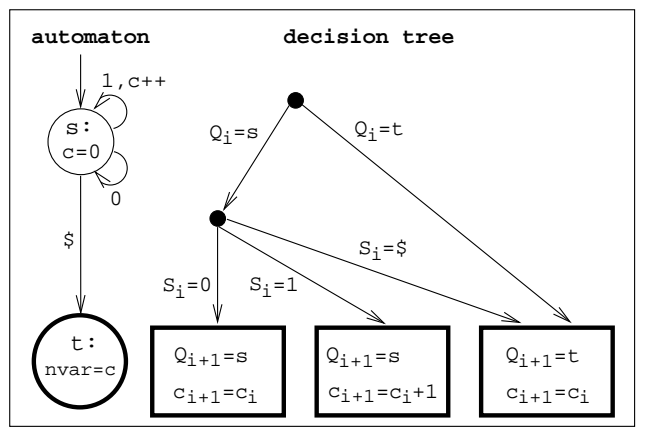

(B)

Fig. 2. Automata and decision trees for $(\mathrm{A}) \leq_{\operatorname{lex}}$ and $(\mathrm{B})$ among.

A couple of examples will help clarify this idea. Note that the decision tree needs to correctly handle the case when the terminal state has already been reached.

Example 2. Consider a $\vec{x} \leq_{\operatorname{lex}} \vec{y}$ constraint over vectors of length $n$. First, we need a signature constraint $\Psi_{\leq_{\text {lex }}}$ relating each pair of arguments $x[i], y[i]$ to a signature variable $S_{i}$. This can be done as follows: $\Psi_{\leq_{\text {lex }}}\left(S_{i}, x[i], y[i]\right) \equiv\left(x[i]<y[i] \Leftrightarrow S_{i}=1\right) \wedge\left(x[i]=y[i] \Leftrightarrow S_{i}=\right.$ 2) $\wedge\left(x[i]>y[i] \Leftrightarrow S_{i}=3\right)$. The automaton of $\leq_{\text {lex }}$ and the decision tree corresponding to the transition constraint $\Phi_{\leq_{\text {lex }}}$ are shown in part (A) of Fig. 2.

Example 3. Consider a among(nvar, vars, values) constraint. First, we need a signature constraint $\Psi_{\text {among }}$ relating each argument vars $[i]$ to a signature letter $S_{i}$. This can be done as follows: $\Psi_{\text {among }}\left(S_{i}, \operatorname{vars}[i]\right.$, values $) \equiv\left(\right.$ vars $[i] \in$ values $\left.\Leftrightarrow S_{i}=1\right) \wedge\left(\right.$ vars $[i] \notin$ values $\left.\Leftrightarrow S_{i}=0\right)$. The automaton of among and the decision tree corresponding to the transition constraint $\Phi_{\text {among }}$ are shown in part (B) of Fig. 2.

Consistency. We consider automata where all subsets of variables in Signature $\mathcal{A}$ rg are pairwise disjoint, and that do not involve counters. Many constraints can be encoded by such automata, for instance the global_contiguity and lex_lesseq constraints presented in Fig. 1. For this kind of automata the filtering algorithm achieves arcconsistency, provided that the filtering algorithms of signature and transition constraints achieve also arc-consistency. To prove this property, consider the constraint hypergraph that represents the conjunction of all signature and transition constraints (see Fig. 3). It has two particular properties: there is no cycle in the corresponding intersection graph ${ }^{2}$,

\footnotetext{
${ }^{2}$ In this graph each vertex corresponds to a constraint and there is an edge between two vertices iff the sets of variables involved in the two corresponding constraints intersect.
} 
and for any pair of constraints the two sets of involved variables share at most one variable. Such an hypergraph is so-called Berge-acyclic [9]. Berge-acyclic constraint

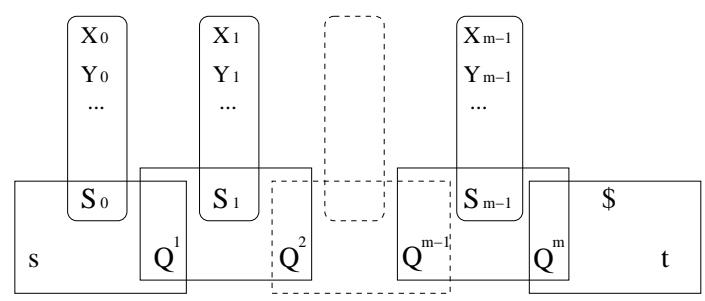

Fig. 3. Constraint hypergraph of the conjunction of transition and signature constraints in the case of disjoint $\mathcal{S}$ ignature $\mathcal{A}$ rg sets. The $i$-th $\mathcal{S}$ ignature $\mathcal{A r g}$ set $\mathcal{S}_{i}$ is denoted by $\left\{X_{i}, Y_{i}, \ldots\right\}$.

networks were proved to be solvable polynomially by achieving arc-consistency [21, 22]. Therefore, if all signature and transition constraints achieve arc-consistency then we obtain a complete filtering for our global constraint.

Performance. It is reasonable to ask the question whether the filtering algorithm described herein performs anywhere near the performance delivered by a hard-coded implementation of a given constraint. To this end, we have compared a version of the Balanced Incomplete Block Design problem [19, prob028] that uses a built-in $\leq_{\text {lex }}$ constraint to break column symmetries with a version using our filtering based on a finite automaton for the same constraint. In a second experiment, we measured the time to find all solutions to a single $\leq_{\operatorname{lex}}$ constraint. The experiments were run in SICStus Prolog 3.11 on a $600 \mathrm{MHz}$ Pentium III. The results are shown in Table 1.

Table 1. Time in seconds for finding (A) the first solution of BIBD instances using built-in vs. simulated $\leq_{\text {lex }}$, and (B) all solutions to a single built-in vs. simulated $\leq_{\text {lex }}$ constraint.

(A)

\begin{tabular}{|l|c|c|}
\hline $\begin{array}{l}\text { Problem } \\
v, b, r, k, \lambda\end{array}$ & Built-in $\leq_{\operatorname{lex}}$ & Simulated $\leq_{\operatorname{lex}}$ \\
\hline $6,50,25,3,10$ & 0.250 & 0.440 \\
\hline $6,60,30,3,12$ & 0.330 & 0.570 \\
\hline $8,14,7,4,3$ & 0.090 & 0.120 \\
\hline $9,120,40,4,10$ & 1.570 & 2.180 \\
\hline $10,90,27,3,6$ & 1.670 & 2.070 \\
\hline $10,120,36,3,8$ & 3.530 & 3.870 \\
\hline $12,88,22,3,4$ & 1.470 & 2.040 \\
\hline $13,104,24,3,4$ & 1.840 & 2.770 \\
\hline $15,70,14,3,2$ & 1.200 & 1.860 \\
\hline
\end{tabular}

(B)

$\begin{gathered}x_{i} \in[0, m-1], y_{i}=m-i \\
|\vec{x}|=|\vec{y}|=m\end{gathered}$
\begin{tabular}{|c|c|c|}
\hline$m$ & Built-in $\leq_{\operatorname{lex}}$ & Simulated $\leq_{\operatorname{lex}}$ \\
\hline 4 & 0.010 & 0.020 \\
\hline 5 & 0.110 & 0.170 \\
\hline 6 & 1.640 & 2.300 \\
\hline 7 & 29.530 & 39.100 \\
\hline
\end{tabular}




\section{Applications of this Technique}

Designing Filtering Algorithm for Global Constraints. We apply this new methodology for designing filtering algorithms for the following fairly large set of global constraints. We came up with an automaton ${ }^{3}$ for the following constraints:

- Unary constraints specifying a domain like in [14] or not_in [17].

- Channeling constraints like domain_constraint [28].

- Counting constraints for constraining the number of occurrences of a given set of values like among [6], at least [17], atmost [17] or count [14].

- Sliding sequence constraints like change [5], longest_change or smooth [3]. longest_change (size, vars, ctr) restricts the variable size to the maximum number of consecutive variables of vars for which the binary constraint $c t r$ holds.

- Variations around the element constraint [20] like element_greatereq [25], element_lesseq [25] or element_sparse [17].

- Variations around the maximum constraint [4] like max_index(vars, index). max_index enforces the variable index to be equal to one of the positions of variables corresponding to the maximum value of the variables of vars.

- Constraints on words like global_contiguity [23], group [17], group_skip_isolated_item [3] or pattern [11].

- Constraints between vectors of variables like between [12], $\leq_{\operatorname{lex}}$ [18], lex_different or differ_from_at_least_k_pos. Given two vectors $\vec{x}$ and $\vec{y}$ which have the same number of components, the constraints lex_different $(\vec{x}, \vec{y})$ and differ_from_at_least_k_pos $(k, \vec{x}, \vec{y})$ respectively enforce the vectors $\vec{x}$ and $\vec{y}$ to differ from at least 1 and $k$ components.

- Constraints between $n$-dimensional boxes like two_quad_are_in_cont act [17] or two_quad_do_not_overlap [7].

- Constraints on the shape of a sequence of variables like inflexion [3], top [8] or valley [8].

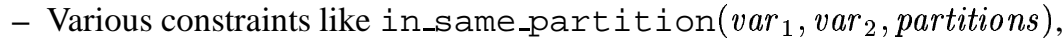
not_all_equal(vars) or sliding_card_skip0(atleast, atmost, vars, values). in_same_partition enforces variables $v a r_{1}$ and $v a r_{2}$ to be respectively assigned to two values that both belong to a same sublist of values of partitions. not_all_equal enforces the variables of vars to take more than a single value. sliding_card_skip0 enforces that each maximum non-zero subsequence of consecutive variables of vars contains at least atleast and atmost values from the set of values values.

Filtering Algorithm for a Conjunction of Global Constraints. Another typical use of our new methodology is to come up with a filtering algorithm for the conjunction of several global constraints. This is usually not easy since this implies analyzing a lot of special cases showing up from the interaction of the different considered constraints. We illustrate this point on the conjunction of the between $(\vec{a}, \vec{x}, \vec{b})$

\footnotetext{
${ }^{3}$ These automata are available in the Appendix. All signature constraints are encoded in order to achieve arc-consistency.
} 
and the exactly_one $(\vec{x}$, values $)$ constraints for which we come with a filtering algorithm, which maintains arc-consistency. The between constraint holds iff $\vec{a} \leq$ lex $\vec{x}$ and $\vec{x} \leq_{\text {lex }} \vec{b}$, while the exactly_one constraint holds if exactly one component of $\vec{x}$ takes its value in the set of values values.

The left-hand part of Fig. 4 depicts the two automata $\mathcal{A}_{1}$ and $\mathcal{A}_{2}$ respectively associated with the between and the exactly_one constraints, while the right-hand part gives the automaton $\mathcal{A}_{3}$ associated with the conjunction of these two constraints. $\mathcal{A}_{3}$ corresponds to the product of $\mathcal{A}_{1}$ and $\mathcal{A}_{2}$. States of $\mathcal{A}_{3}$ are labeled by the two states of $\mathcal{A}_{1}$ and $\mathcal{A}_{2}$ they were issued. Transitions of $\mathcal{A}_{3}$ are labeled by the end symbol $\$$ or by a conjunction of elementary conditions, where each condition is taken in one of the following set of conditions $\left\{a_{i}<x_{i}, a_{i}=x_{i}, a_{i}>x_{i}\right\},\left\{b_{i}>x_{i}, b_{i}=x_{i}, b_{i}<x_{i}\right\}$, $\left\{x_{i} \in\right.$ values, $x_{i} \notin$ values $\}$. This makes up to $3 \cdot 3 \cdot 2=18$ possible combinations and leads to the signature constraint $\Psi_{\text {between^exactly_one }}\left(S_{i}, a_{i}, x_{i}, b_{i}\right.$, values $)$ between the signature variable $S_{i}$ and the $i$-th component of vectors $\vec{a}, \vec{x}$ and $\vec{b}$ :

$$
S_{i}= \begin{cases}0 & \text { if } a_{i}<x_{i} \wedge b_{i}>x_{i} \wedge x_{i} \notin \text { values, } \quad 9 \text { if } a_{i}<x_{i} \wedge b_{i}>x_{i} \wedge x_{i} \in \text { values } \\ 1 & \text { if } a_{i}<x_{i} \wedge b_{i}=x_{i} \wedge x_{i} \notin \text { values, } 10 \text { if } a_{i}<x_{i} \wedge b_{i}=x_{i} \wedge x_{i} \in \text { values } \\ 2 & \text { if } a_{i}<x_{i} \wedge b_{i}<x_{i} \wedge x_{i} \notin \text { values, } 11 \text { if } a_{i}<x_{i} \wedge b_{i}<x_{i} \wedge x_{i} \in \text { values }, \\ 3 & \text { if } a_{i}=x_{i} \wedge b_{i}>x_{i} \wedge x_{i} \notin \text { values, } 12 \text { if } a_{i}=x_{i} \wedge b_{i}>x_{i} \wedge x_{i} \in \text { values } \\ 4 & \text { if } a_{i}=x_{i} \wedge b_{i}=x_{i} \wedge x_{i} \notin \text { values, } 13 \text { if } a_{i}=x_{i} \wedge b_{i}=x_{i} \wedge x_{i} \in \text { values } \\ 5 & \text { if } a_{i}=x_{i} \wedge b_{i}<x_{i} \wedge x_{i} \notin \text { values, } 14 \text { if } a_{i}=x_{i} \wedge b_{i}<x_{i} \wedge x_{i} \in \text { values } \\ 6 & \text { if } a_{i}>x_{i} \wedge b_{i}>x_{i} \wedge x_{i} \notin \text { values, } 15 \text { if } a_{i}>x_{i} \wedge b_{i}>x_{i} \wedge x_{i} \in \text { values } \\ 7 & \text { if } a_{i}>x_{i} \wedge b_{i}=x_{i} \wedge x_{i} \notin \text { values, } 16 \text { if } a_{i}>x_{i} \wedge b_{i}=x_{i} \wedge x_{i} \in \text { values } \\ 8 & \text { if } a_{i}>x_{i} \wedge b_{i}<x_{i} \wedge x_{i} \notin \text { values, } 17 \text { if } a_{i}>x_{i} \wedge b_{i}<x_{i} \wedge x_{i} \in \text { values }\end{cases}
$$

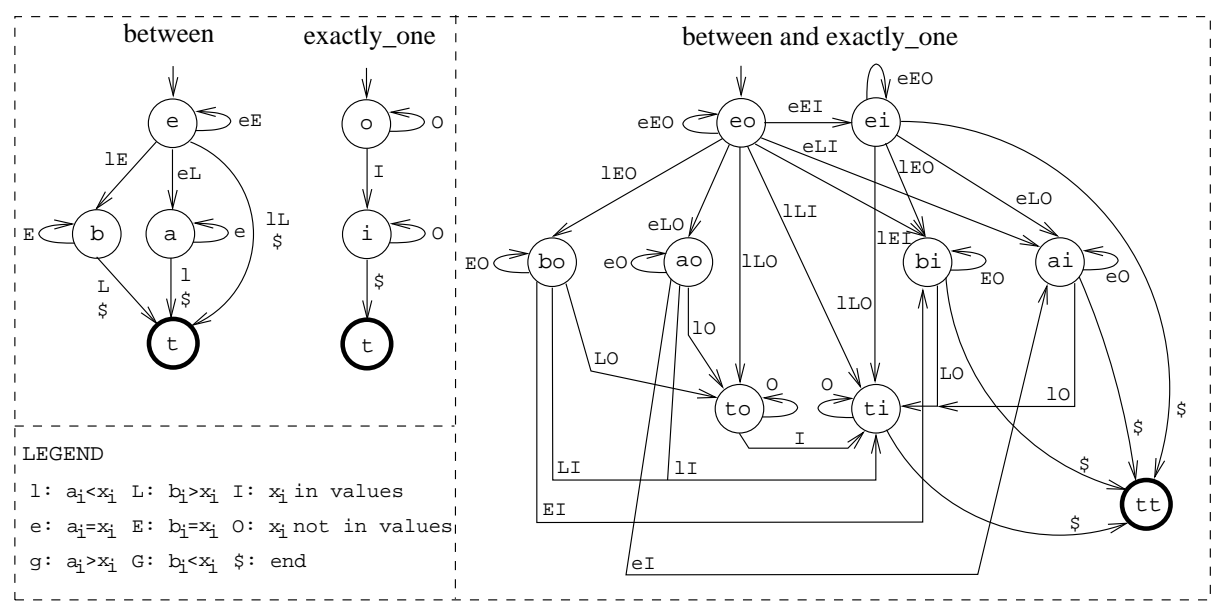

Fig. 4. Automata associated with between and exactly_one and the automaton associated with their conjunction.

In order to achieve arc-consistency on the conjunction of the between $(\vec{a}, \vec{x}, \vec{b})$ and the exactly_one $(\vec{x}$, values $)$ constraints we need to have arc-consistency on 
$\Psi_{\text {between^exactly_one }}\left(S_{i}, a_{i}, x_{i}, b_{i}\right.$, values $)$. In our context this is done by using the global constraint programming facilities of SICStus Prolog [14] ${ }^{4}$

Example 4. Consider three variables $x \in\{0,1\}, y \in\{0,3\}, z \in\{0,1,2,3\}$ subject to the conjunction of constraints between $(\langle 0,3,1\rangle,\langle x, y, z\rangle,\langle 1,0,2\rangle) \wedge$ exact ly_one $(\langle x, y, z\rangle,\{0\})$. Even if both the between and the exact ly_one constraints achieve arc-consistency, we need the automaton associated with their conjunction to find out that $z \neq 0$. This can be seen as follows: after two transitions, the automaton will be either in state a $i$ or in state bi. However, in either state, a 0 must already have been seen, and so there is no support for $z=0$.

Constraints Involving Finite State Systems. A third use of our methodology is for handling problems involving finite state systems where the transition diagram is known. In this context the variables of the global constraint correspond to the sequence of triggered transitions (i.e. the signature variables) and the constraint enforces ending up in a terminal state of the diagram. Let us consider the problem of the man, wolf, goat and cabbage problem to illustrate this idea.

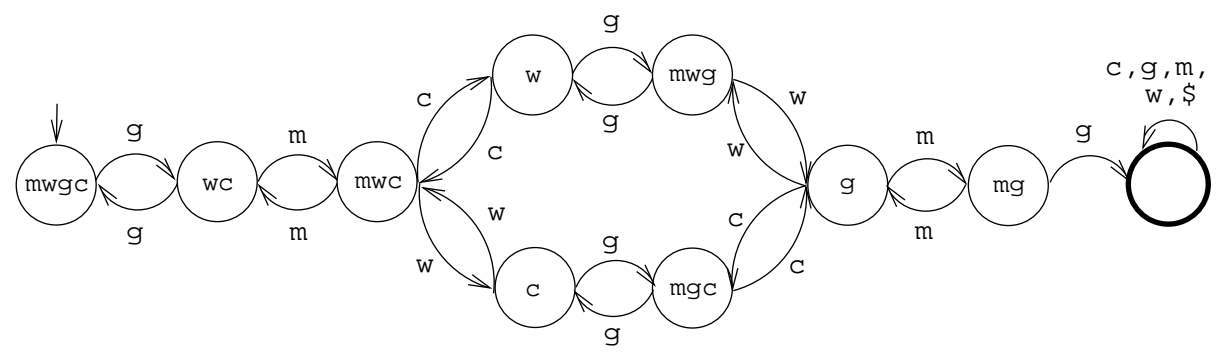

Fig. 5. Automata associated to the man, wolf, goat and cabbage problem.

Example 5. A man, a wolf, a goat and a cabbage are on the bank of a river. They want to cross to the other bank, and the man can ferry each across, one at a time. However both the wolf and the goat as well as the goat and the cabbage should not be left unattended. Fig. 5 gives the automaton associated to this problem. Each state is labeled by the subset that is on the left bank, while each transition is labeled by the action the man takes (e.g. $m$ for cross alone, $w$ for cross with the wolf, $g$ for cross whith the goat, $c$ for cross with the cabbage). On the final state, the loop models the fact that once we have reached the final state we are done.

\section{Handling Relaxation for a Counter-Free Automaton}

This section presents a filtering algorithm for handling constraint relaxation under the hypothesis that we don't use any counter in our automaton. It can be seen as a generalization of the algorithm used for the regular constraint [26].

Definition 1. The violation cost of a global constraint is the minimum number of subsets of its signature argument for which it is necessary to change at least one variable in order to get back to a solution.

\footnotetext{
${ }^{4}$ The corresponding code is available in the Appendix..
} 
When these subsets form a partition over the variables of the constraint and when they consist of a single element, this cost is in fact the minimum number of variables to unassign in order to get back to a solution. As in [27], we add a cost variable cost as an extra argument of the constraint. Our filtering algorithm first evaluates the minimum cost value $\mathcal{M}$ in. Then, according to $\max (\operatorname{cost})$, it prunes values that cannot belong to a solution.

Example 6. Consider the constraint global_contiguity $\left(\left[V_{0}, V_{1}, V_{2}, V_{3}, V_{4}, V_{5}, V_{6}\right]\right)$ with the following current domains for variables $V_{i}$ : $[\{0,1\},\{1\},\{1\},\{0\},\{1\},\{0,1\},\{1\}]$. The constraint is violated because there are necessarily at least two distinct sequences of consecutive 1. To get back to a state that can lead to a solution, it is enough to turn the fourth value to 1 . One can deduce $\mathcal{M}$ in $=1$. Consider now the relaxed form soft_global_contiguity $\left(\left[V_{0}, V_{1}, V_{2}, V_{3}, V_{4}, V_{5}, V_{6}\right]\right.$, cost $)$ and assume $\max ($ cost $)=1$. The filtering algorithm should remove value 0 from $V_{5}$. Indeed, selecting value 0 for variable $V_{5}$ entails a minimum violation cost of 2 . Observe that for this constraint the signature variables $S_{0}, S_{1}, S_{2}, S_{3}, S_{4}, S_{5}, S_{6}$ are $V_{0}, V_{1}, V_{2}, V_{3}, V_{4}, V_{5}, V_{6}$.

As in the algorithm of Pesant [26], our consistency algorithm builds a layered acyclic directed multigraph $\mathcal{G}$. Each layer of $\mathcal{G}$ contains a different node for each state of our automaton. Arcs only appear between consecutive layers. Given two nodes $n_{1}$ and $n_{2}$ of two consecutive layers, $q_{1}$ and $q_{2}$ denote their respective associated state. There is an $\operatorname{arc} a$ from $n_{1}$ to $n_{2}$ iff, in the automaton, there is an $\operatorname{arc} \operatorname{arc}\left(q_{1}, v, q_{2}\right)$ from $q_{1}$ to $q_{2}$. The arc $a$ is labeled with the value $v$. Arcs corresponding to transitions that cannot be triggered according to the current domain of the signature variables $S_{0}, \ldots, S_{m-1}$ are marked as infeasible. All other arcs are marked as feasible. Finally, we discard isolated nodes from our layered multigraph. Since our automaton has a single initial state and a single final state, $\mathcal{G}$ has one source and one sink respectively denoted by source and sink.

Example 6 continued. Part (A) of Fig. 6 recalls the automaton of the global_contiguity constraint, while part (B) gives the multigraph $\mathcal{G}$ associated with the soft_global_contiguity constraint previously introduced. Each arc is labeled by the condition associated to its corresponding transition. Each node contains the name of the corresponding automaton state. Numbers in a node will be explained later on. Infeasible arcs are represented with a dotted line.

We now explain how to use the multigraph $\mathcal{G}$ to evaluate the minimum violation cost $\mathcal{M i n}$ and to prune the signature variables according to the maximum allowed violation cost $\max ($ cost $)$.

Evaluating the minimum violation cost $\mathcal{M}$ in can be seen as finding the path from the source to the sink of $\mathcal{G}$ that contains the smallest number of infeasible arcs. This can be done by performing a topological sort starting from the source of $\mathcal{G}$. While performing the topological sort, we compute for each node $n_{k}$ of $\mathcal{G}$ the minimum number of infeasible arcs from the source of $\mathcal{G}$ to $n_{k}$. This number is recorded in before $\left[n_{k}\right]$. At the end of the topological sort, the minimum violation cost $\mathcal{M i n}$ we search for, is equal to before $[\operatorname{sink}]$.

Notation 1 Let $i$ be assignable to a signature variable $S_{l}$. Min $n_{l}^{i}$ denotes the minimum violation cost value according to the hypothesis that we assign $i$ to $S_{l}$. 


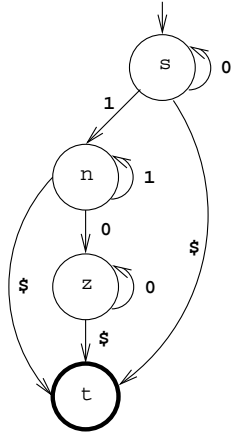

(A) Automaton for global_contiguity

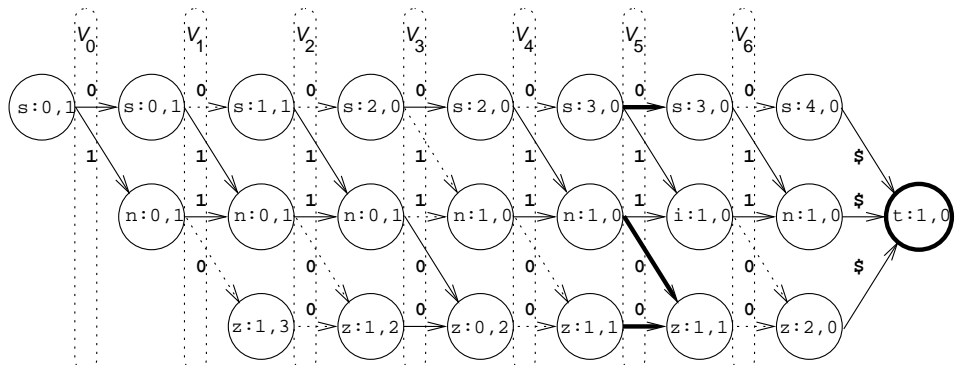

(B) Graph of potential executions of the automaton of global_contiguity according to $V_{0}, V_{1}, V_{2}, V_{3}, V_{4}, V_{5}, V_{6}$

Fig. 6. Relaxing the global_contiguity constraint.

To prune domains of signature variables we need to compute the quantity $\mathcal{M i n}_{l}^{i}$. In order to do so, we introduce the quantity after $\left[n_{k}\right]$ for a node $n_{k}$ of $\mathcal{G}$ : after $\left[n_{k}\right]$ is the minimum number of infeasible arcs on all paths from $n_{k}$ to sink. It is computed by performing a second topological sort starting from the sink of $\mathcal{G}$. Let $\mathcal{A}_{l}^{i}$ denote the set of arcs of $\mathcal{G}$, labeled by $i$, for which the origin has a rank of $l$. The quantity $\min _{a \rightarrow b \in \mathcal{A}_{l}^{i}}$ (before $[a]+$ after $\left.[b]\right)$ represents the minimum violation cost under the hypothesis that $S_{l}$ remains assigned to $i$. If that quantity is greater than $\mathcal{M} i n$ then there is no path from source to sink which uses an arc of $\mathcal{A}_{l}^{i}$ and which has a number of infeasible arcs equal to $\mathcal{M i n}$. In that case the smallest cost we can achieve is $\mathcal{M i n}+1$. Therefore we have:

$$
\mathcal{M i n} n_{l}^{i}=\min \left(\min _{a \rightarrow b \in \mathcal{A}_{l}^{i}}(\text { before }[a]+\operatorname{after}[b]), \mathcal{M} i n+1\right)
$$

The filtering algorithm is then based on the following theorem:

Theorem 1. Let $i$ be a value from the domain of a signature variable $S_{l}$. If $\mathcal{M i n}_{l}^{i}>$ $\max ($ cost $)$ then $i$ can be removed from $S_{l}$.

The cost of the filtering algorithm is dominated by the two topological sorts. They have a cost proportional to the number of arcs of $\mathcal{G}$ which is bounded by the number of signature variables times the number of arcs of the automaton.

Example 6 continued. Let us come back to the instance of Fig. 6. Beside the state's name, each node $n_{k}$ of part (B) of Fig. 6 gives the values of before $\left[n_{k}\right]$ and of after $\left[n_{k}\right]$. Since before $[\operatorname{sink}]=1$ we have that the minimum cost violation is equal to 1 . Pruning can be potentially done only for signature variables having more than one value. In our example this corresponds to variables $V_{0}$ and $V_{5}$. So we evaluate the four quantities $\mathcal{M} i n_{0}^{0}=\min (0+1,2)=1$, $\mathcal{M i n}_{0}^{1}=\min (0+1,2)=1, \mathcal{M i n}_{5}^{0}=\min (\min (3+0,1+1,1+1), 2)=2, \mathcal{M} i n_{5}^{1}=$ $\min (\min (3+0,1+0), 2)=1$. If $\max ($ cost $)$ is equal to 1 we can remove value 0 from $V_{5}$. The corresponding arcs are depicted with a thick line in Fig. 6. 


\section{Conclusion and Perspectives}

The automaton description introduced in this reportcan be seen as a restricted programming language. This language is used for writing down a constraint checker, which verifies whether a ground instance of a constraint is satisfied or not. This checker allows pruning the variables of a non-ground instance of a constraint by simulating all potential executions of the corresponding program according to the current domain of the variables of the relevant constraint. This simulation is achieved by encoding all potential executions of the automaton as a conjunction of signature and transition constraints and by letting the usual constraint propagation deducing all the relevant information. We want to stress the key points of this approach:

- Within the context of global constraints, it was implicitly assumed that providing a constraint checker is a much easier task than coming up with a filtering algorithm. It was also commonly admitted that the design of filtering algorithms is a difficult task which involves creativity and which cannot be automatized. We have shown that this is not the case any more if one can afford to provide a constraint checker.

- Non-determinism has played a key role by augmenting programming languages with backtracking facilities [16], which was the origin of logic programming. Nondeterminism also has a key role to play in the systematic design of filtering algorithms: finding a filtering algorithm can be seen as the task of executing in a nondeterministic way the deterministic program corresponding to a constraint checker and to extract the relevant information which for sure occurs under any circumstances. This can indeed be achieved by using constraint programming.

We finally present different perspectives of this work.

- A natural continuation would be to extend the automaton description in order to get closer to a classical imperative programming language. This would allow reusing directly available checkers in order to systematically get a filtering algorithm.

- Another interesting question is about identifying other structural conditions on the signature and transition constraints that can guarantee arc-consistency for the original global constraint.

\section{Acknowledgements}

We are grateful to Irit Katriel for suggesting us the use of topological sort for the relaxation part, and to Christian Bessière for his helpful comments with respect to Bergeacyclic CSP's.

\section{References}

1. A.G.Frutos, Q.Liu, A.J.Thiel, A.M.W.Sanner, A.E.Condon, L.M.Smith, and R.M.Corn. Demonstration of a word design strategy for DNA computing on surfaces. Nucleic Acids Research, 25:4748-4757, 1997. 
2. J. Amilhastre, H. Fargier, and P. Marquis. Consistency restoration and explanations in dynamic CSPs - application to configuration. Artificial Intelligence, 135:199-234, 2002.

3. N. Beldiceanu. Global constraints as graph properties on structured network of elementary constaints of the same type. In R. Dechter, editor, CP'2000, Principles and Practice of Constraint Programming, volume 1894 of LNCS, pages 52-66. Springer-Verlag, 2000.

4. N. Beldiceanu. Pruning for the minimum constraint family and for the number of distinct values constraint family. In T. Walsh, editor, CP'2001, Int. Conf. on Principles and Practice of Constraint Programming, volume 2239 of LNCS, pages 211-224. Springer-Verlag, 2001.

5. N. Beldiceanu and M. Carlsson. Revisiting the cardinality operator and introducing the cardinality-path constraint family. In P. Codognet, editor, ICLP'2001, Int. Conf. on Logic Programming, volume 2237 of LNCS, pages 59-73. Springer-Verlag, 2001.

6. N. Beldiceanu and E. Contejean. Introducing global constraints in CHIP. Mathl. Comput. Modelling, 20(12):97-123, 1994.

7. N. Beldiceanu, Q. Guo, and S. Thiel. Non-overlapping constraints between convex polytopes. In T. Walsh, editor, Principles and Practice of Constraint Programming (CP'2001), volume 2239 of LNCS, pages 392-407. Springer-Verlag, 2001.

8. N. Beldiceanu and E. Poder. Cumulated profiles of minimum and maximum resource utilisation. In Ninth Int. Conf. on Project Management and Scheduling, 2004.

9. C. Berge. Graphs and hypergraphs. Dunod, Paris, 1970.

10. B. Boigelot and P. Wolper. Representing arithmetic constraints with finite automata: An overview. In Peter J. Stuckey, editor, ICLP'2002, Int. Conf. on Logic Programming, volume 2401 of $L N C S$, pages 1-19. Springer-Verlag, 2002.

11. S. Bourdais, P. Galinier, and G. Pesant. HIBISCUS: A constraint programming application to staff scheduling in health care. In F. Rossi, editor, CP'2003, Principles and Practice of Constraint Programming, volume 2833 of LNCS, pages 153-167. Springer-Verlag, 2003.

12. M. Carlsson and N. Beldiceanu. From constraints to finite automata to filtering algorithms. In D. Schmidt, editor, Proc. ESOP2004, volume 2986 of LNCS, pages 94-108. SpringerVerlag, 2004.

13. M. Carlsson et al. SICStus Prolog User's Manual. Swedish Institute of Computer Science, 3.11 edition, January 2004. http: //www. sics. se/sicstus/.

14. M. Carlsson, G. Ottosson, and B. Carlson. An open-ended finite domain constraint solver. In H. Glaser, P. Hartel, and H. Kuchen, editors, Programming Languages: Implementations, Logics, and Programming, volume 1292 of LNCS, pages 191-206. Springer-Verlag, 1997.

15. C.Flamm, I.L.Hofacker, and P.F.Stadler. RNA in silico: The computational biology of RNA secondary structures. Adv. Complex Syst., 2:5-90, 1999.

16. J. Cohen. Non-deterministic algorithms. ACM Computing Surveys, 11(2):79-94, 1979.

17. COSYTEC. CHIP Reference Manual, v5 edition, 2003.

18. A. Frisch, B. Hnich, Z. Kizıltan, I. Miguel, and T. Walsh. Global constraints for lexicographic orderings. In Pascal Van Hentenryck, editor, Principles and Practice of Constraint Programming (CP'2002), volume 2470 of LNCS, pages 93-108. Springer-Verlag, 2002.

19. I.P. Gent and T. Walsh. CSPLib: a benchmark library for constraints. Technical Report APES-09-1999, APES, 1999. http: / / www . csplib.org.

20. P. Van Hentenryck and J.-P. Carillon. Generality vs. specificity: an experience with AI and OR techniques. In National Conference on Artificial Intelligence (AAAI-88), 1988.

21. P. Janssen and M-C. Vilarem. Problèmes de satisfaction de contraintes: techniques de résolution et application à la synthèse de peptides. Research Report C.R.I.M., 54, 1988.

22. P. Jégou. Contribution à l'étude des problèmes de satisfaction de contraintes: algorithmes de propagation et de résolution. Propagation de contraintes dans les réseaux dynamiques. $P h D$ Thesis, 1991.

23. M. Maher. Analysis of a global contiguity constraint. In Workshop on Symmetry on RuleBased Constraint Reasoning and Programming, 2002. held along CP-2002. 
24. M. Milano. Constraint and integer programming. Kluwer Academic Publishers, 2004. ISBN 1-4020-7583-9.

25. G. Ottosson, E. Thorsteinsson, and J. N. Hooker. Mixed global constraints and inference in hybrid IP-CLP solvers. In CP'99 Post-Conference Workshop on Large-Scale Combinatorial Optimization and Constraints, pages 57-78, 1999.

26. G. Pesant. A regular language membership constraint for sequence of variables. In Workshop on Modelling and Reformulation Constraint Satisfaction Problems, pages 110-119, 2003.

27. T. Petit, J.-C. Régin, and C. Bessière. Specific filtering algorithms for over-constrained problems. In T. Walsh, editor, Principles and Practice of Constraint Programming (CP'2001), volume 2239 of $L N C S$, pages 451-463. Springer-Verlag, 2001.

28. P. Refalo. Linear formulation of constraint programming models and hybrid solvers. In R. Dechter, editor, Principles and Practice of Constraint Programming (CP'2000), volume 1894 of LNCS, pages 369-383. Springer-Verlag, 2000.

29. N. R. Vempaty. Solving constraint satisfaction problems using finite state automata. In $\mathrm{Na}$ tional Conference on Artificial Intelligence (AAAI-92), pages 453-458. AAAI Press, 1992. 


\section{A List of Constraints and their Corresponding Automata}

\section{A.1 among (NVAR, VARIABLES, VALUES) [6]}

NVAR is the number of variables of the collection VARIABLES which take their value in VALUES.

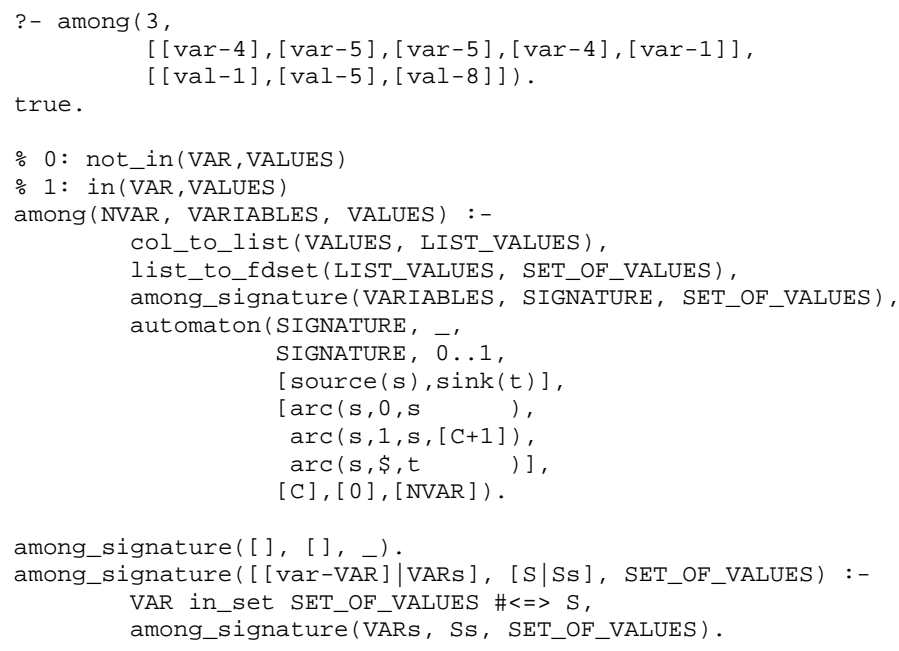

\section{A.2 at least (N, VARIABLES, VALUE) [17]}

At least $N$ variables of the VARIABLES collection are assigned to value VALUE.

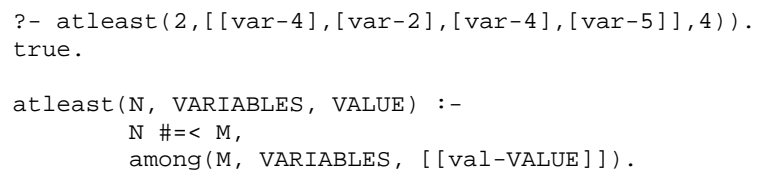

\section{A.3 atmost (N, VARIABLES, VALUE) [17]}

At most $\mathrm{N}$ variables of the VARIABLES collection are assigned to value VALUE.

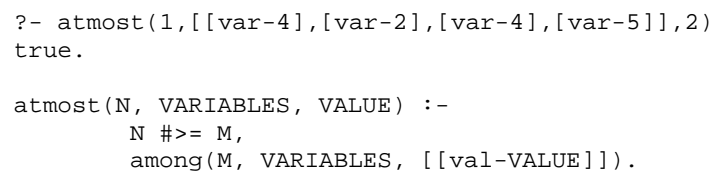

\section{A.4 between (As, Xs, Bs) [12]}

Vector $\mathrm{X}$ is lexicographically greater than or equal to vector $\mathrm{A}$ and vector $\mathrm{X}$ is lexicographically less than or equal to vector B. 


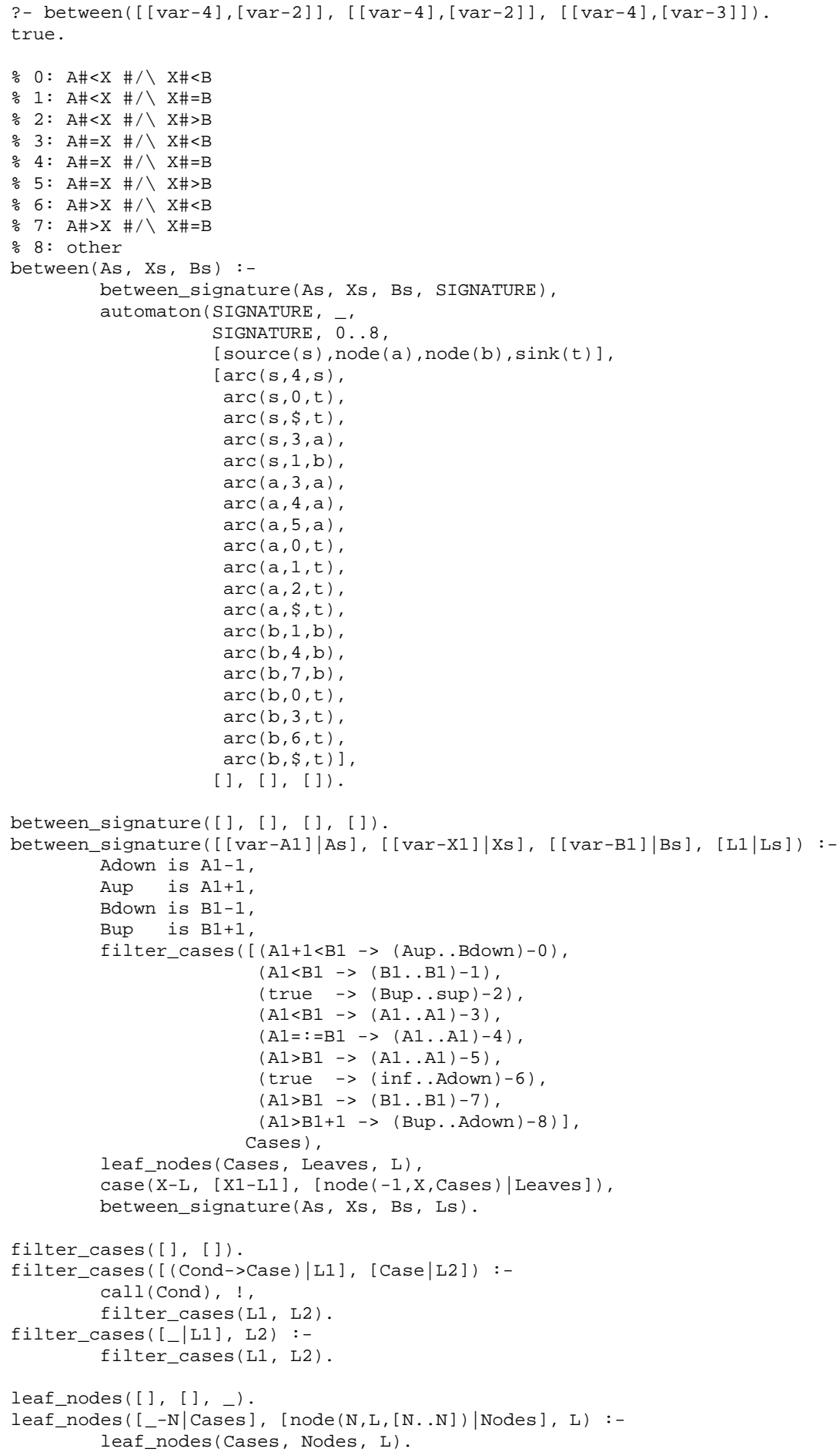




\section{A.5 between_exactly_one (As, Xs, Bs, VALUES)}

Vector Xs is lexicographically greater than or equal to vector As and vector Xs is lexicographically less than or equal to
vector Bs and exactly one component of vector Xs has a value in the set of values VALUES.

?- between_exactly_one([[var-4], [var-2]], [[var-4], [var-2]], [[var-4], [var-3]], $[[\operatorname{val}-1],[\operatorname{val}-2],[\operatorname{val}-6]])$.

true.

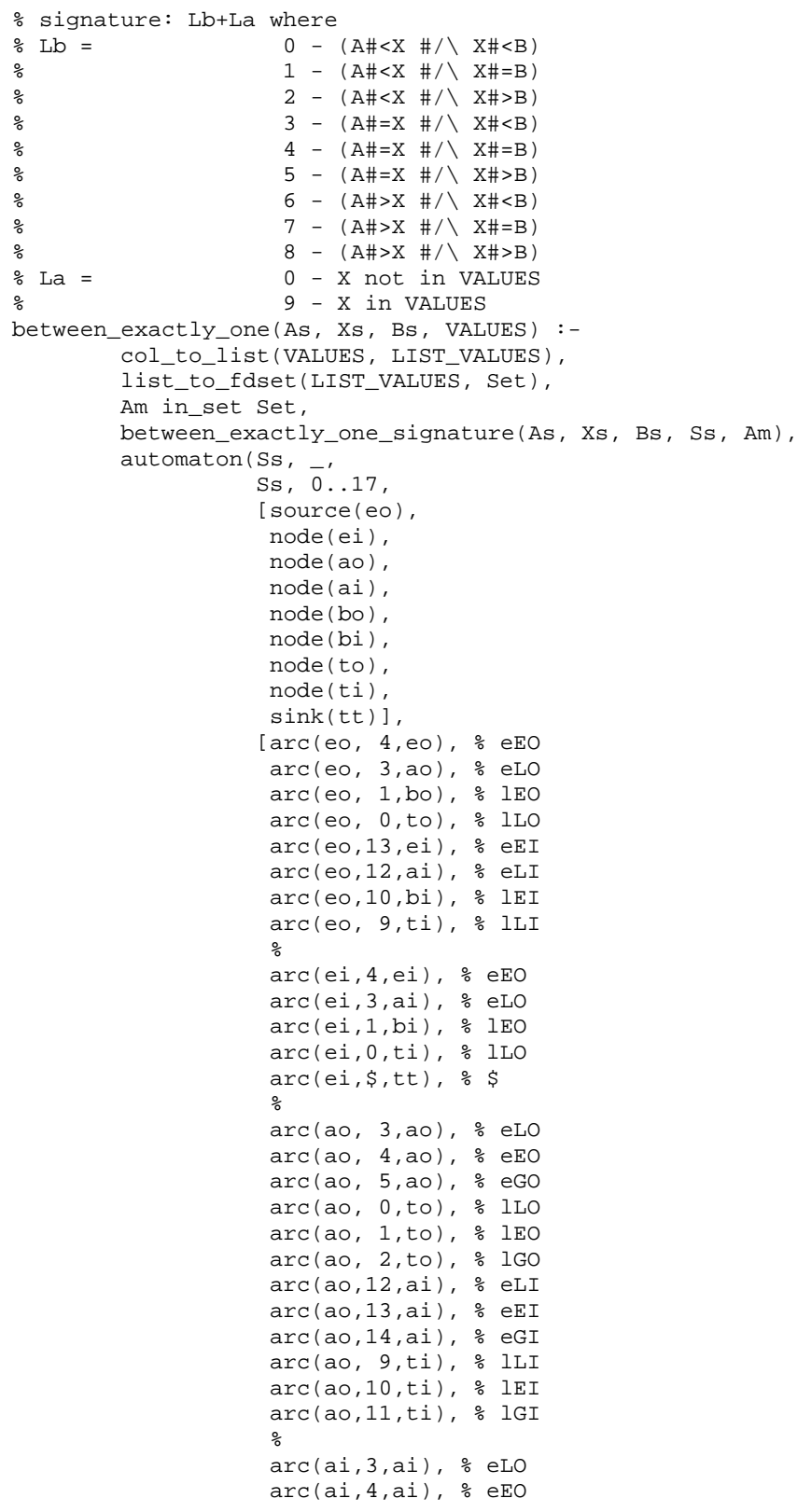




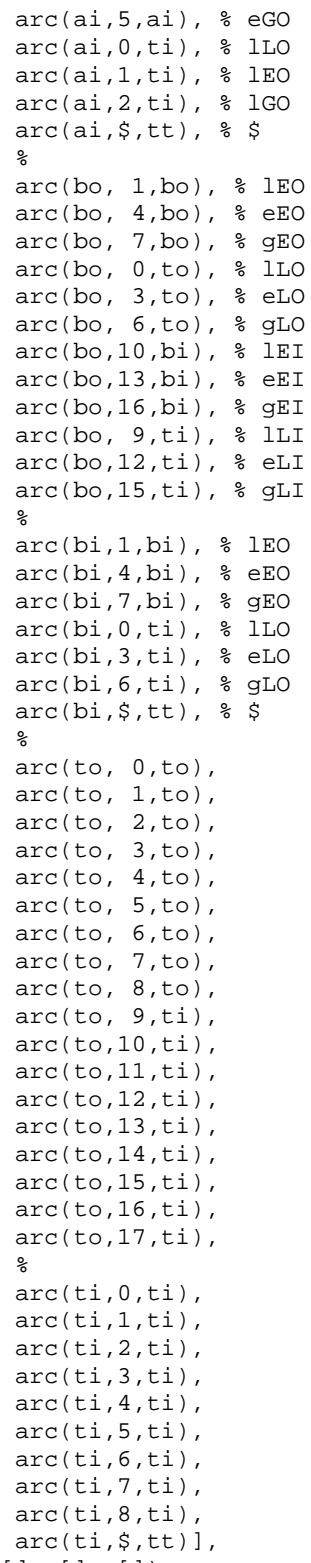




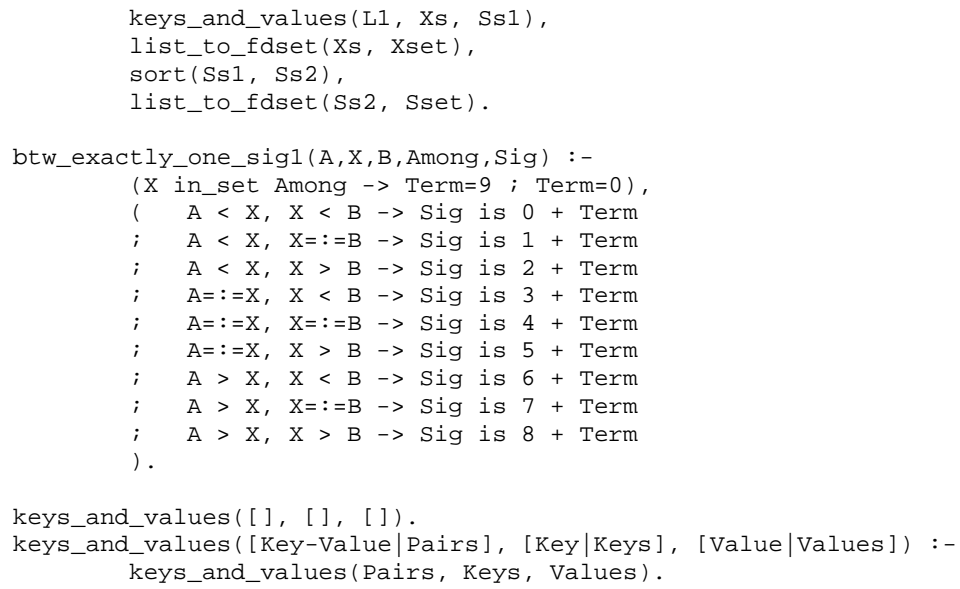

\section{A.6 change (NCHANGE, VARIABLES, CTR) $[17,5]$}

NCHANGE is the number of times that constraint CTR holds on consecutive variables of the collection VARIABLES.

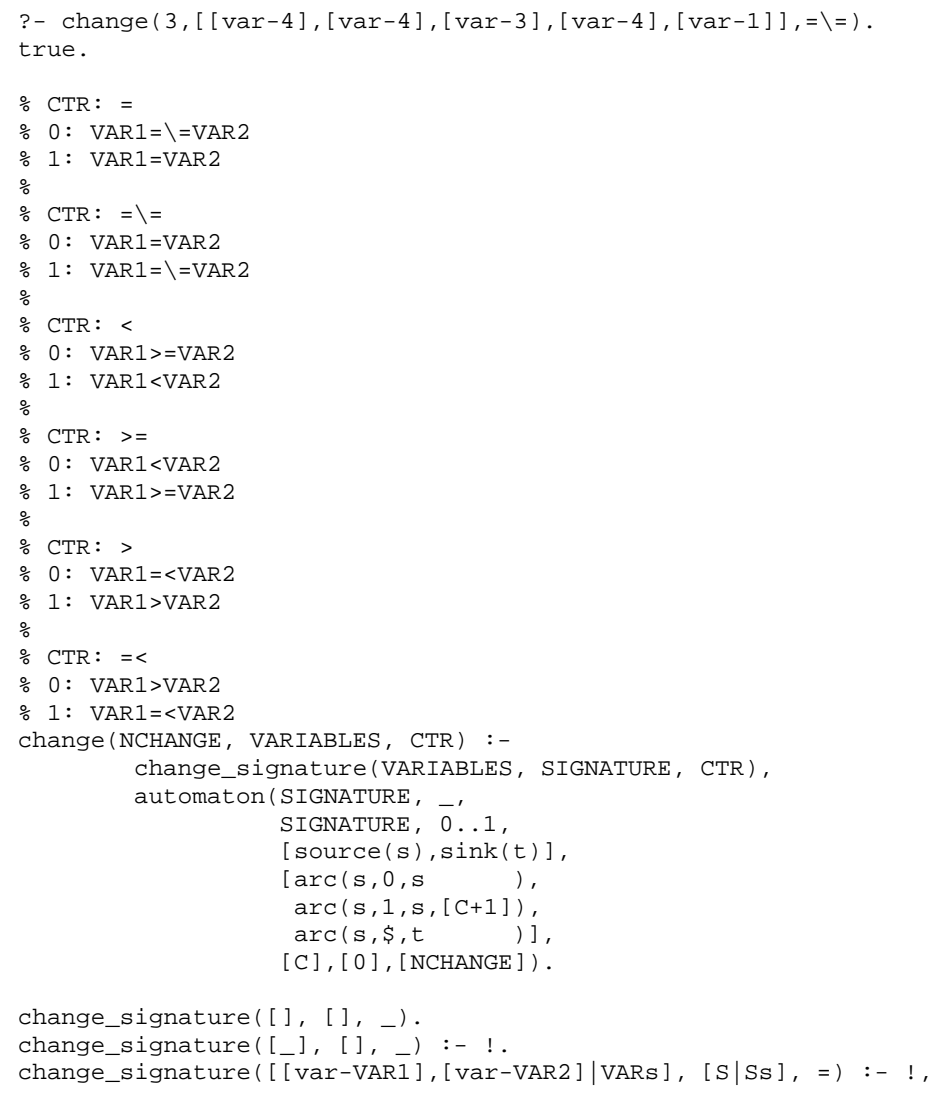




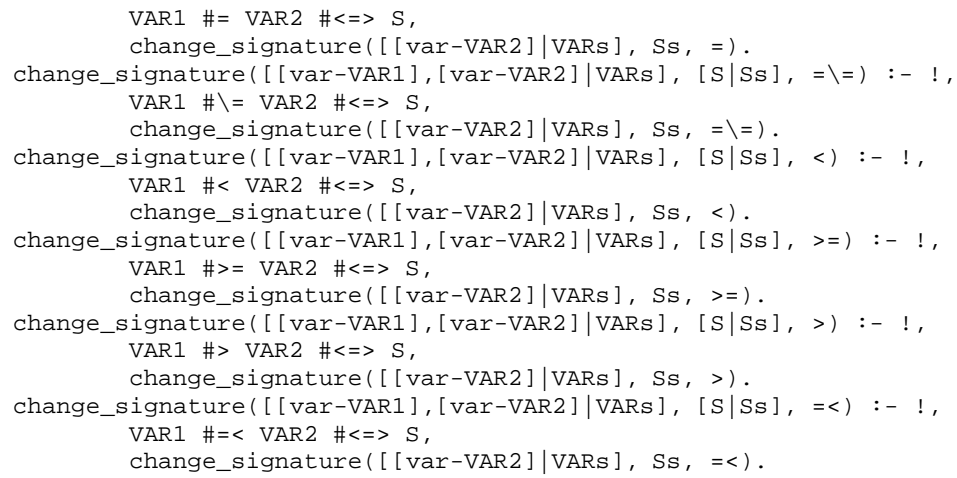

\section{A.7 circular_change (NCHANGE, VARIABLES, CTR)}

NCHANGE is the number of times that CTR holds on consecutive variables of the collection VARIABLES. The last and the first variables of the collection VARIABLES are also considered to be consecutive.

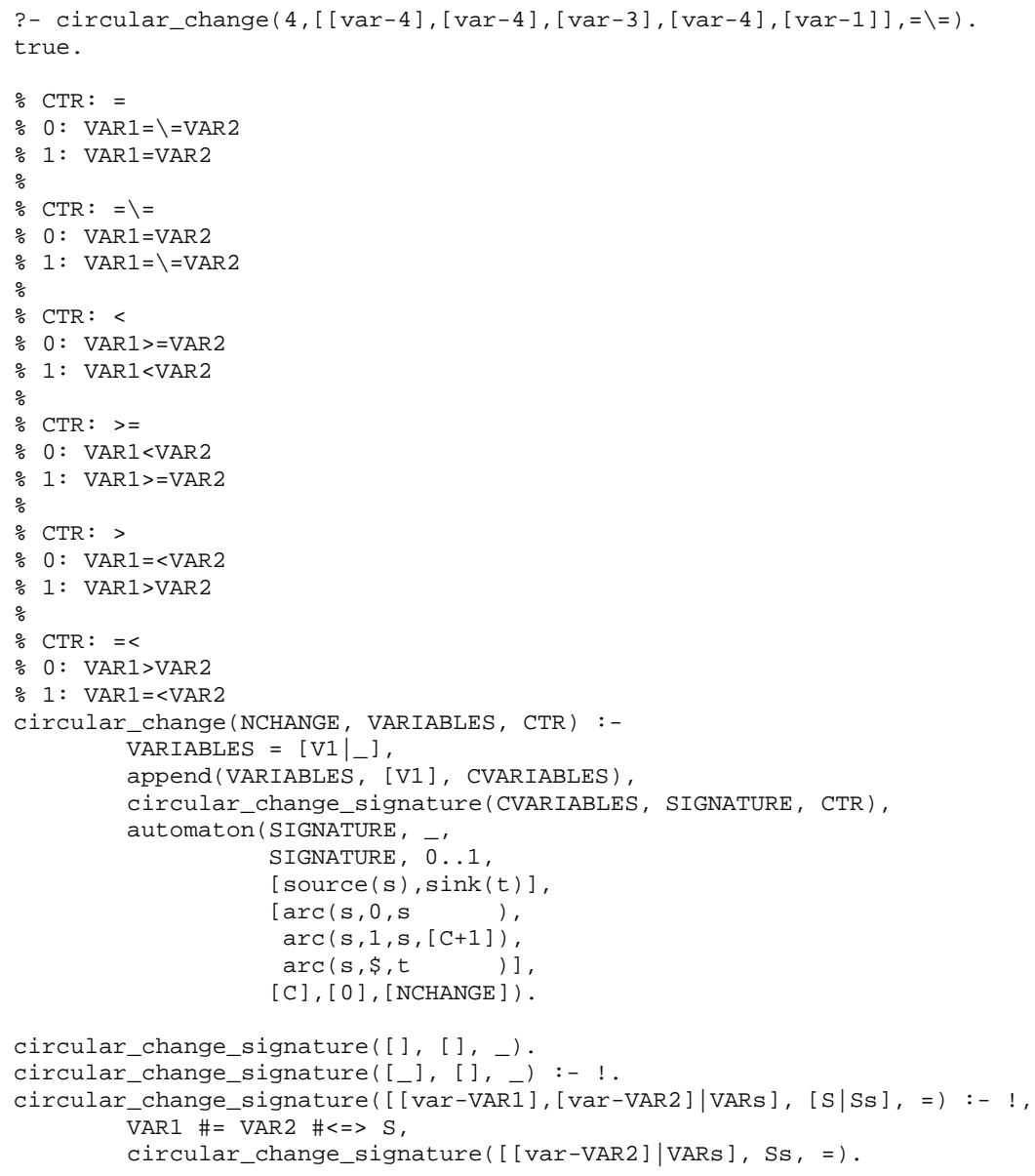




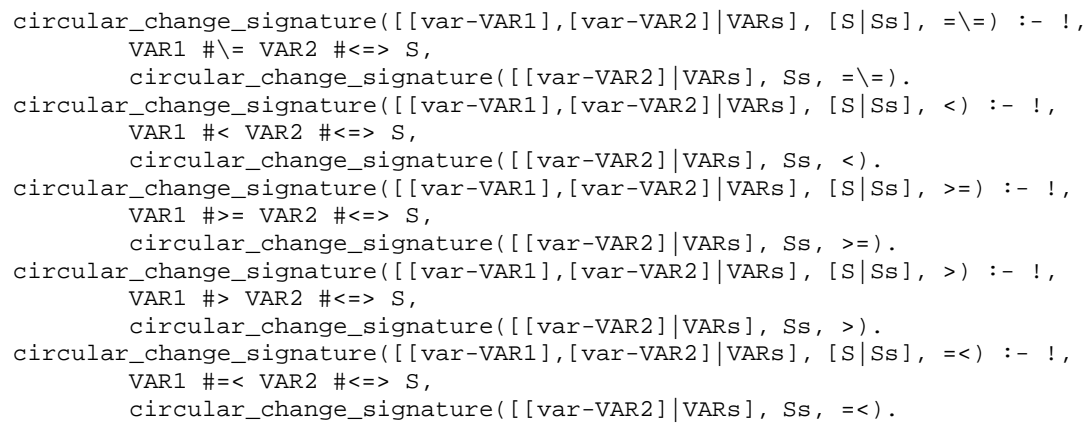

\section{A.8 count_(VALUE, VARIABLES, RELOP, NVAR) [13]}

Let $\mathrm{N}$ be the number of variables of the VARIABLES collection assigned to value VAL; Enforce condition N RELOP NVAR to hold.

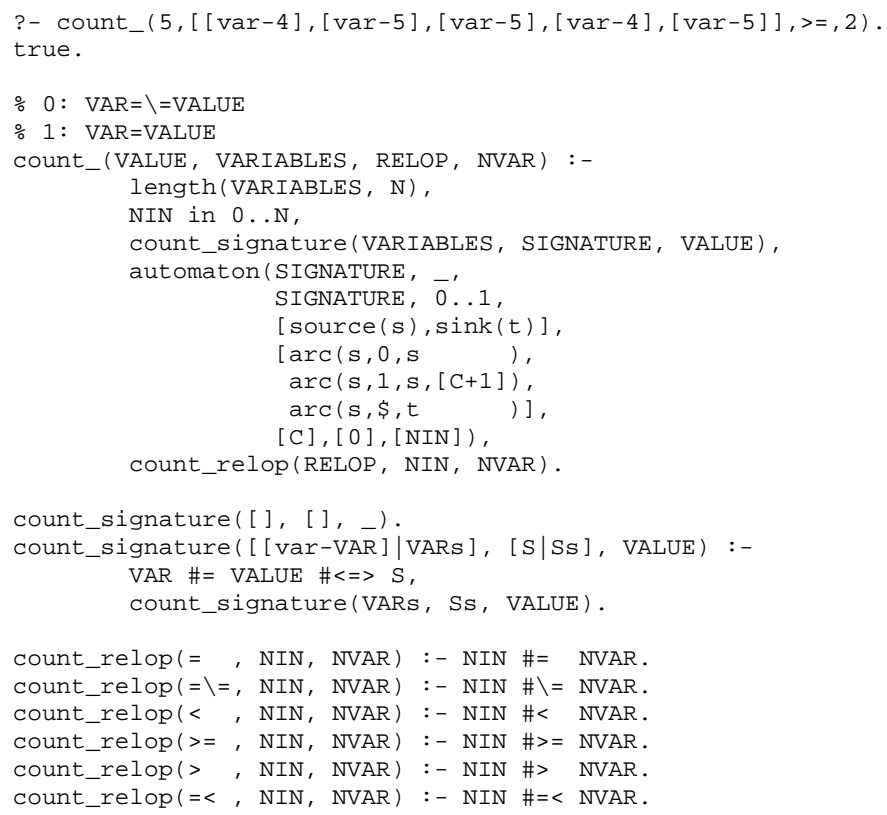

\section{A.9 counts (VALUES, VARIABLES, RELOP, LIMIT)}

Let $\mathrm{N}$ be the number of variables of the VARIABLES collection assigned to a value of the VALUES collection. Enforce condition N RELOP LIMIT to hold. Inspired by count.

?- counts([[val-1], [val-3], [val-4], [val-9]],

true.

$[[\operatorname{var}-4],[\operatorname{var}-5],[\operatorname{var}-5],[\operatorname{var}-4],[\operatorname{var}-1],[\operatorname{var}-5]],=, 3)$.

\% : not_in (VAR, VALUES)

\% 1 : in (VAR, VALUES)

counts (VALUES, VARIABLES, RELOP, LIMIT) :- 
length (VARIABLES, N),

NIN in $0 \ldots N$,

col to list (VALUES, LIST VALUES)

list_to_fdset (LIST_VALUES, SET_OF_VALUES),

counts_signature (VARIABLES, SIGNATURE, SET_OF_VALUES),

automaton (SIGNATURE,

SIGNATURE, 0 ..1,

$[\operatorname{source}(s), \operatorname{sink}(t)]$,

$[\operatorname{arc}(s, 0, s)$,

$\operatorname{arc}(s, \$, t)]$

$[\mathrm{C}],[0],[N I N])$,

count_relop (RELOP, NIN, LIMIT).

counts_signature ([], [], _).

counts_signature([ [var-VAR] $\mid \mathrm{VARS}],[\mathrm{S} \mid \mathrm{SS}], \mathrm{SET}-\mathrm{OF} \_$VALUES) :-

VAR in_set SET_OF_VALUES \#<=> $\mathrm{S}$,

counts_signature(VARs, Ss, SET_OF_VALUES).

\section{A.10 deepest_valley (DEPTH, VARIABLES)}

DEPTH is the maximum value of the quantity $\min \left(V_{i}, V_{k}\right)-V_{j}$ where $V_{i}, V_{j}, V_{k}$ are three variables of the VARIABLES collection such that $i<j<k, V_{i}>V_{j}$ and $V_{j}<V_{k}$. If no such variables exists DEPTH is equal to 0 .

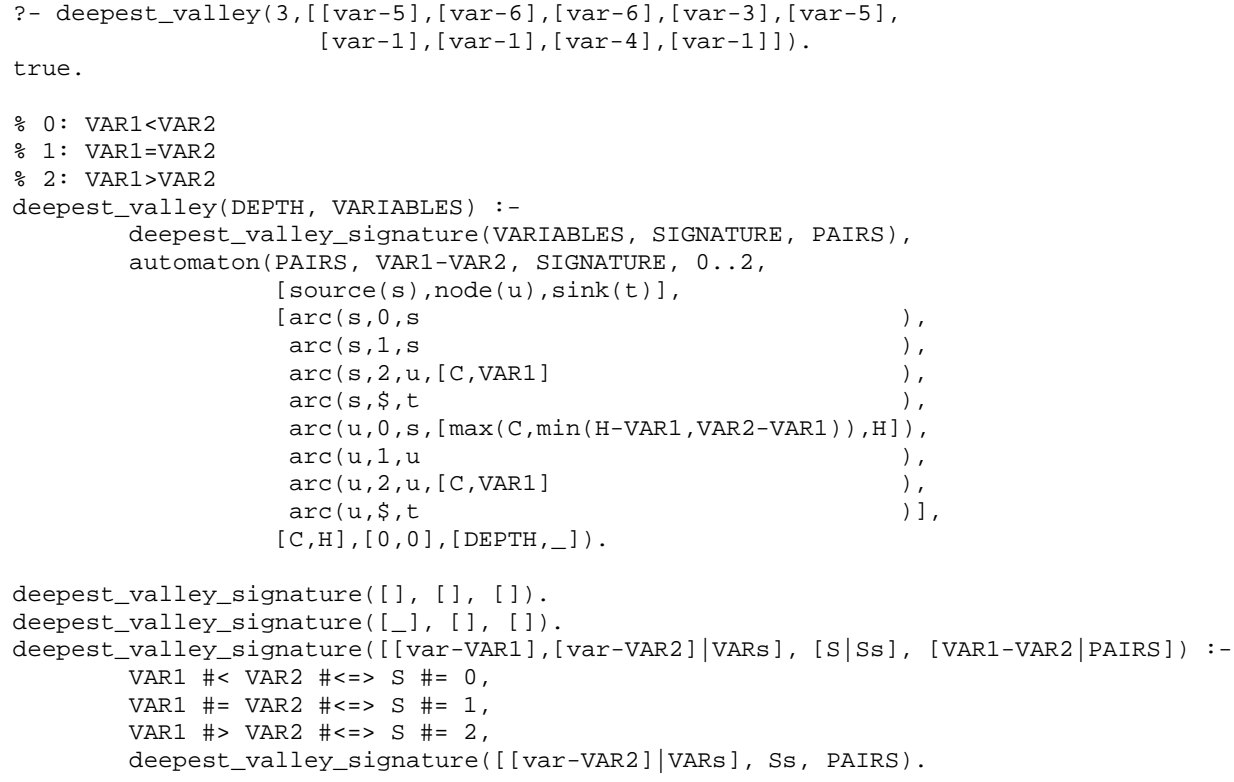

\section{A.11 differ_from_at_least_k_pos (K,VECTOR1, VECTOR2)}

Enforce two vectors VECTOR1 and VECTOR2 to differ from at least K positions. Inspired by [1].

?- differ_from_at_least_k_pos(3,[[var-2],[var-5],[var-2],[var-0]],

true.

$[[\operatorname{var}-3],[\operatorname{var}-6],[\operatorname{var}-2],[\operatorname{var}-1]])$.

$\therefore 0: \quad X=\backslash=Y$ 


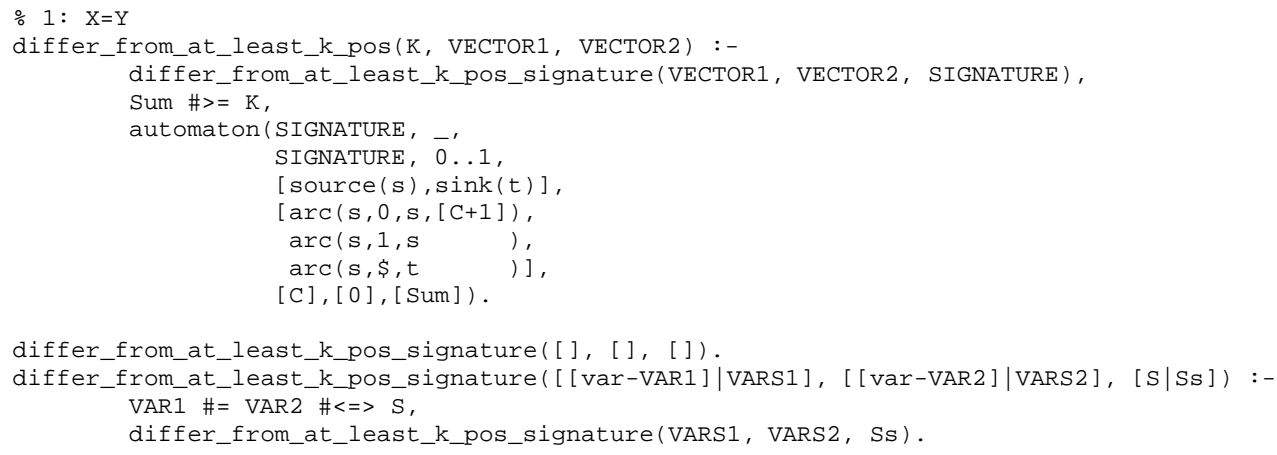

\section{A.12 domain_constraint (VAR, VALUES) [28]}

Make the link between a domain variable VAR and those $0-1$ variables that are associated to each potential value of VAR: The $0-1$ variable associated to the value taken by variable VAR is equal to 1 , while the remaining $0-1$ variables are all equal to 0 .

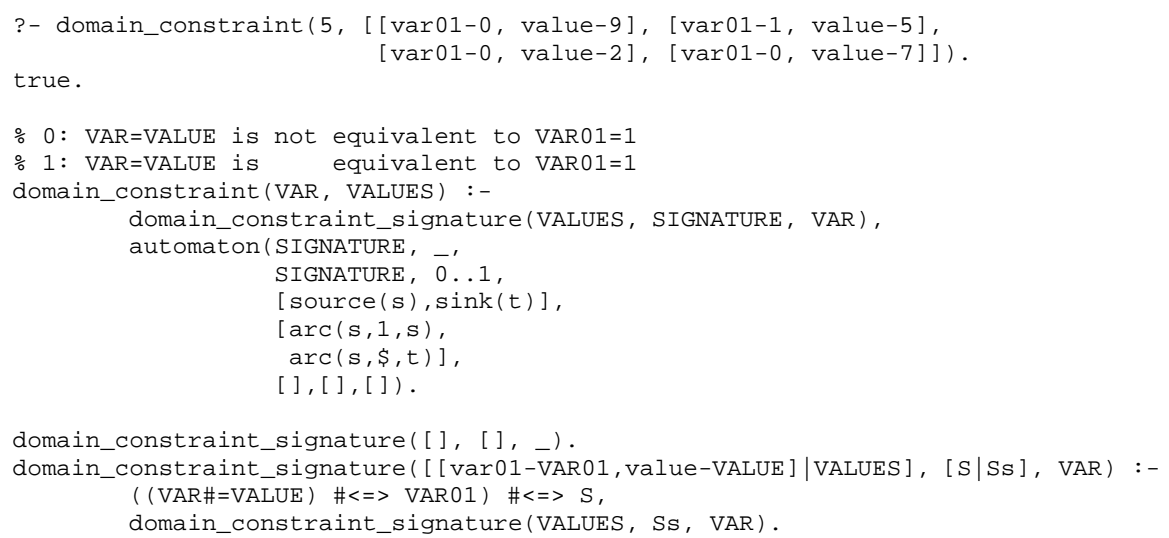

\section{A.13 elem (ITEM, TABLE)}

ITEM is equal to one of the entries of the table TABLE.

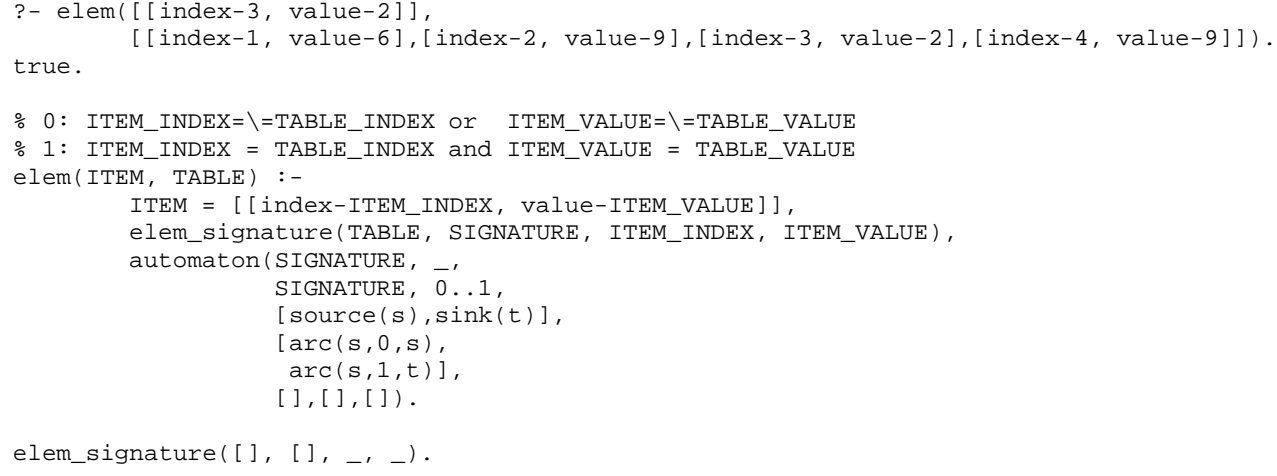




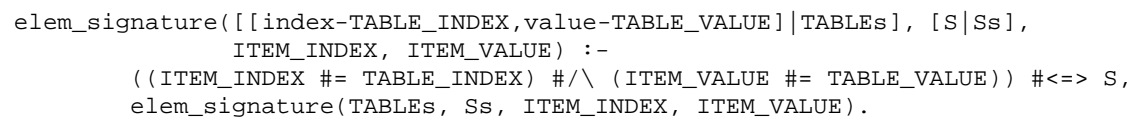

\section{A.14 element_(INDEX, TABLE, VALUE) [20]}

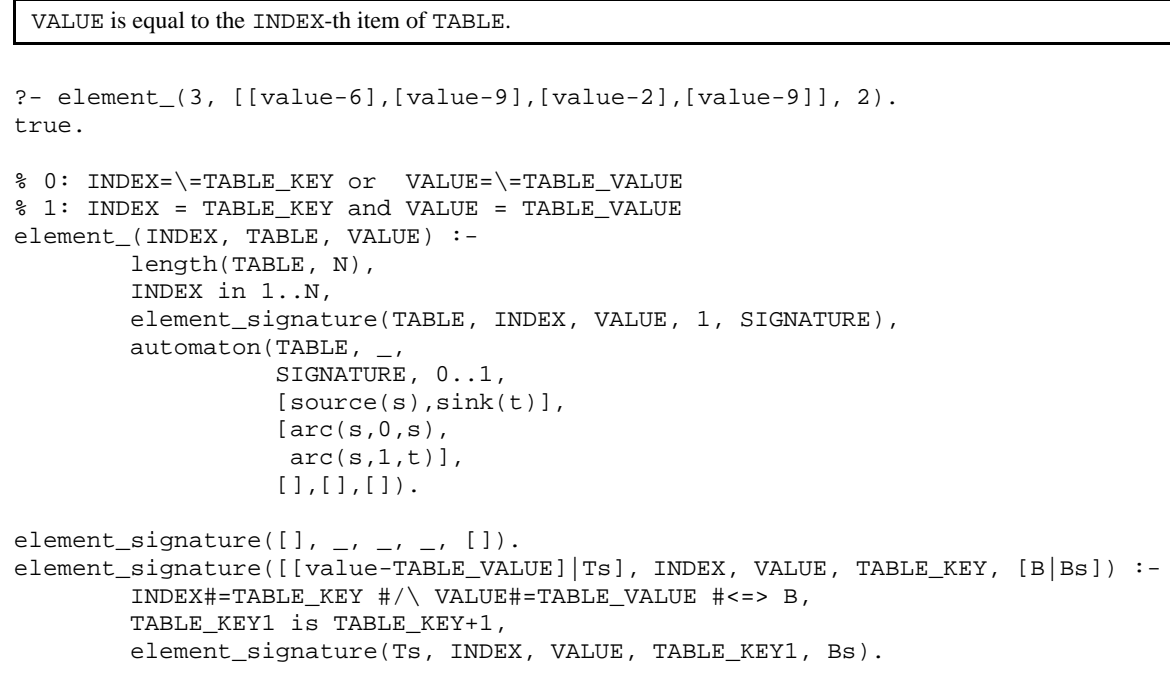

\section{A.15 element_greatereq (ITEM, TABLE) [25]}

ITEM.value is greater than or equal to one of the entries (i.e. the value attribute) of the table TABLE.

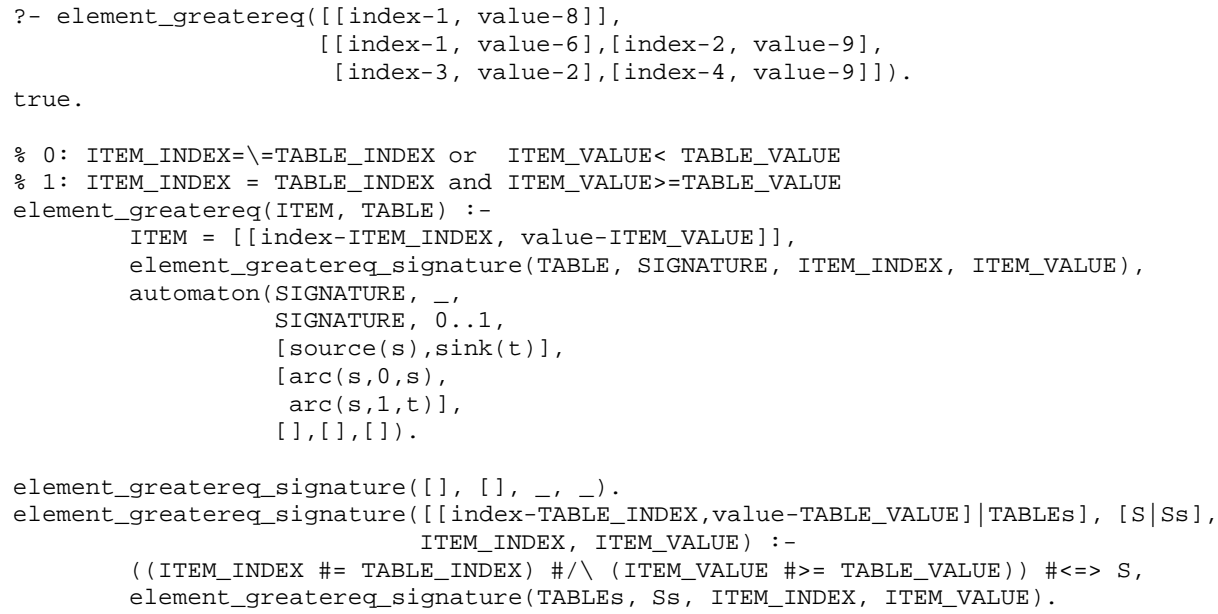

\section{A.16 element_lesseq(ITEM, TABLE) [25]}

ITEM.value is less than or equal to one of the entries (i.e. the value attribute) of the table TABLE. 


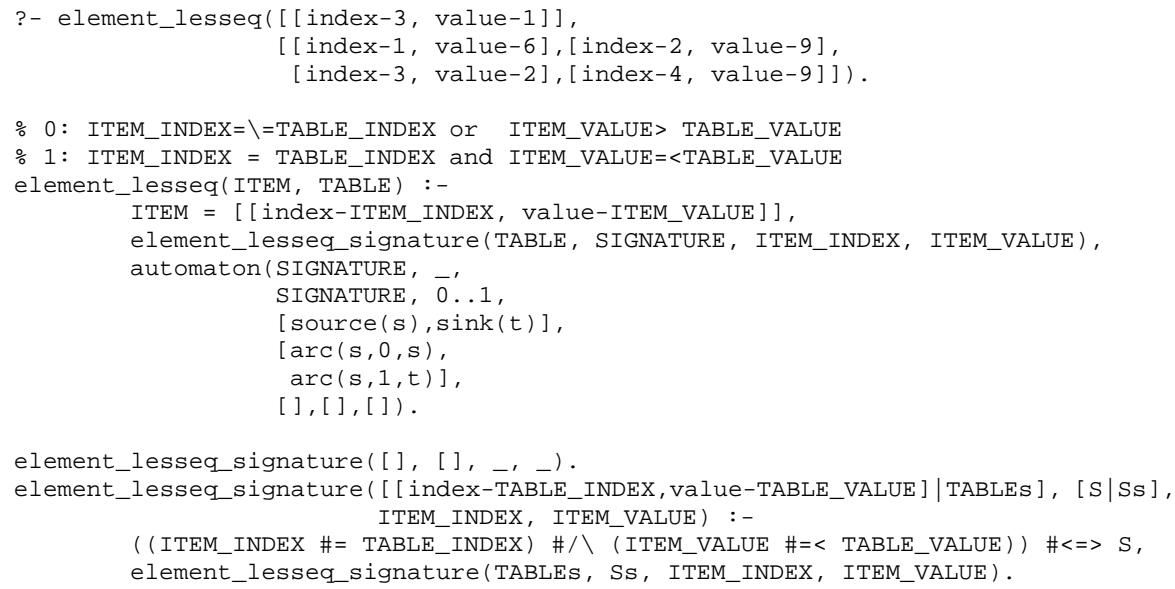

\section{A.17 element_sparse (ITEM, TABLE, DEFAULT) [17]}

ITEM. value is equal to one of the entries of the table TABLE or to the default value DEFAULT if the entry ITEM. index does not exist in TABLE.

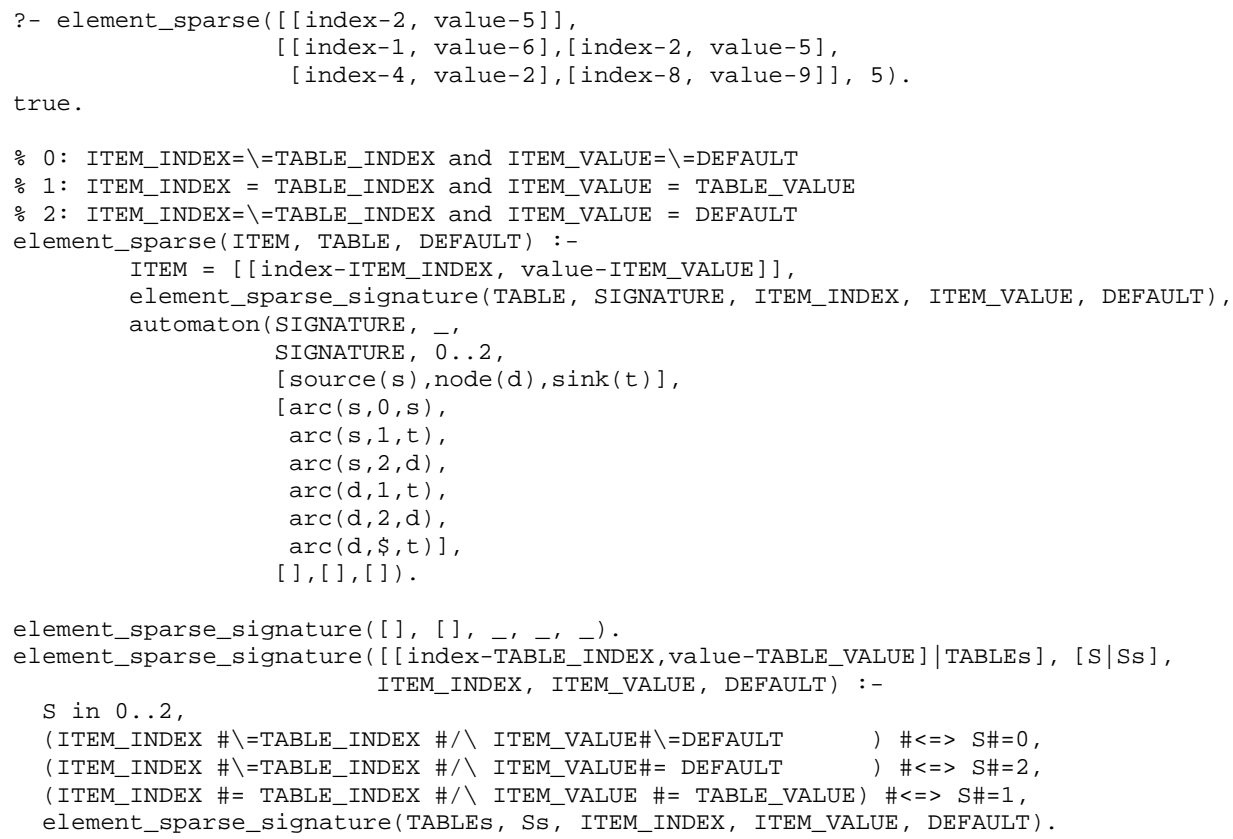

\section{A.18 global_contiguity (VARIABLES) [23]}

Enforce all variables of the VARIABLES collection to be assigned to 0 or 1 . In addition, all variables assigned to value 1 appear contiguously. 


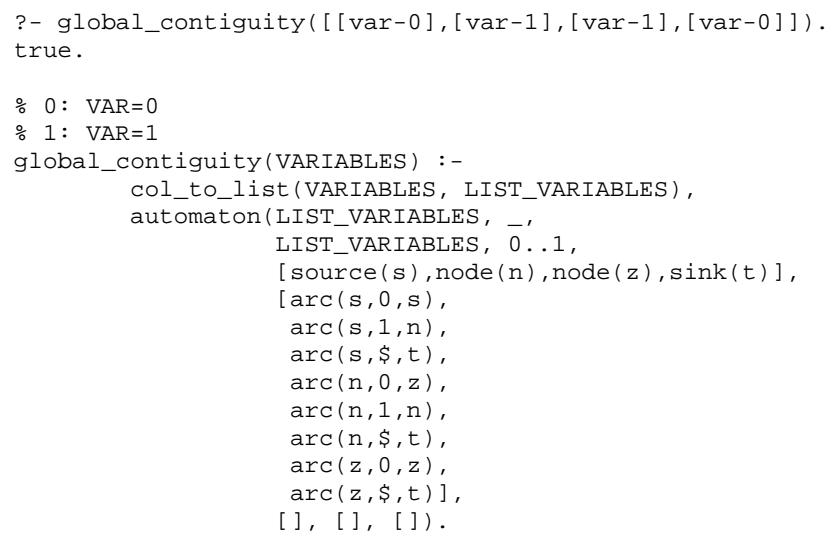

\section{A.19 group (MIN_SIZE, MAX_SIZE, NGROUP, VARIABLES, VALUES) [17]}

Let $n$ be the number of variables of the collection VARIABLES. Let $X_{i}, X_{i+1}, \ldots, X_{j}(1 \leq i \leq j \leq n)$ be consecutive variables of the collection of variables VARIABLES such that all the following conditions simultaneously apply:

- All variables $X_{i}, \ldots, X_{j}$ take their value in the set of values VALUES,

- $i=1$ or $X_{i-1}$ does not take a value in VALUES,

- $j=n$ or $X_{j+1}$ does not take a value in VALUES.

We call such a set of variables a group. The constraint group is true if all the following conditions hold:

- There are exactly NGROUP groups of variables,

- MIN_SIZE is the number of variables of the smallest group,

- MAX_SIZE is the number of variables of the largest group.

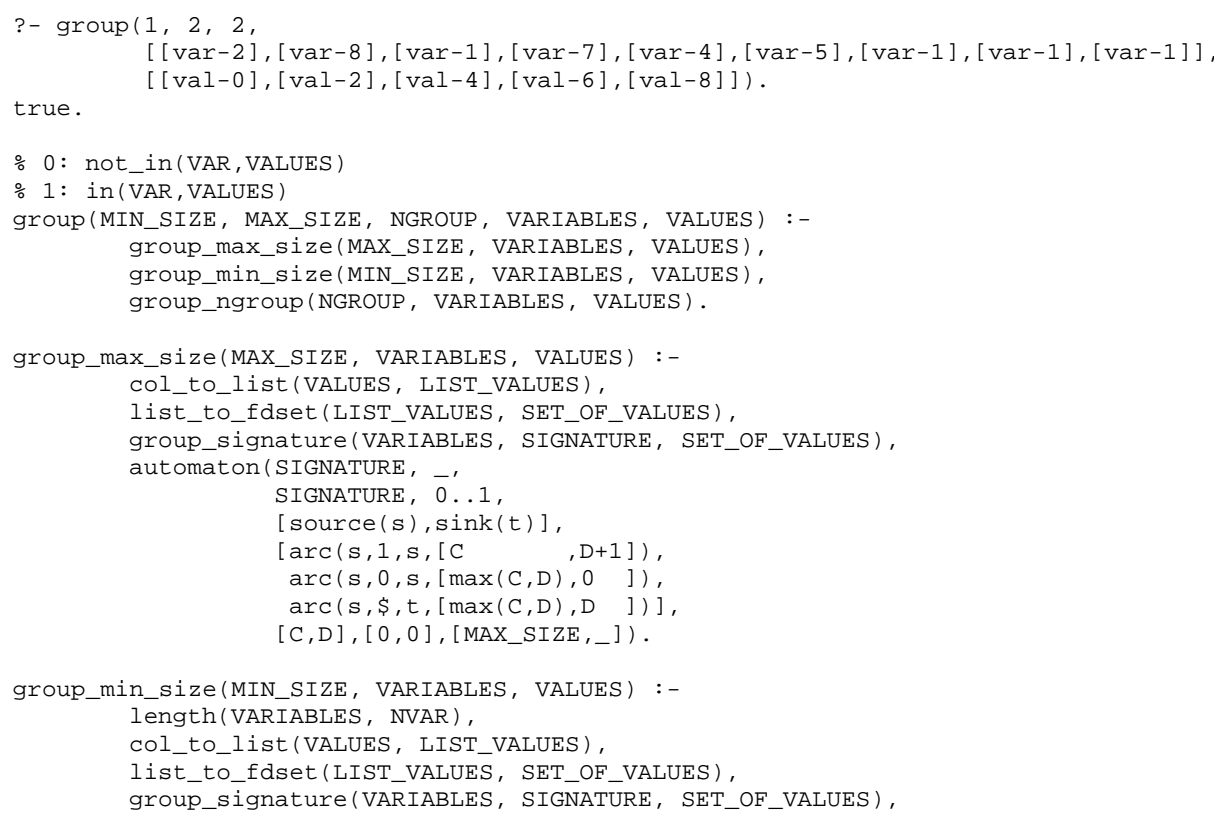


automaton (SIGNATURE, -,

SIGNATURE, $0 \ldots 1$,

[source $(s), \operatorname{node}(j), \operatorname{node}(k), \operatorname{sink}(t)]$,

$[\operatorname{arc}(s, 0, s$

$\operatorname{arc}(s, s, t$

, D I),

$\operatorname{arc}(j, 1, j,[c$

$, D+1])$,

$\operatorname{arc}(j, 0, k,[\min (C, D), D])$,

$\operatorname{arc}(j, s, t,[\min (C, D), D])$,

$\operatorname{arc}(\mathrm{k}, 0, \mathrm{k}$

,

$\operatorname{arc}(k, 1, j,[\mathrm{C}, 1])$,

$\operatorname{arc}(k, \$, t,[\min (C, D), D])]$,

$[C, D],[0,1],[$ MIN_SIZE,_]).

group_ngroup (NGROUP, VARIABLES, VALUES) :-

Col_to_list(VALUES, LIST_VALUES),

list_to_fdset (LIST_VALUES, SET_OF_VALUES)

group_signature(VARIABLES, SIGNATURE, SET_OF_VALUES),

automaton (SIGNATURE,

SIGNATURE, $\overline{0} \ldots 1$,

source $(s), \operatorname{node}(i), \operatorname{sink}(t)]$

$[\operatorname{arc}(s, 0, s$

),

$\operatorname{arc}(s, 1, i,[C+1])$,

$\operatorname{arc}(s, \$, t)$

$\operatorname{arc}(i, 1, i$

$\operatorname{arc}(i, 0$,

$\operatorname{arc}(i, \$, t \quad)]$

$[\mathrm{C}],[0],[\mathrm{NGROUP}])$.

group_signature([], [],_).

group_signature([[Var-VAR] $\mid$ VARs $],$ [S|SS $],$ SET_OF_VALUES) :-

VAR in_set SET_OF_VALUES \#<=> S,

group_signature (VARs, Ss, SET_OF_VALUES).

\section{A.20 group_skip_isolated_item (MIN_SIZE, MAX_SIZE, NGROUP , VARIABLES, VALUES)}

Let $n$ be the number of variables of the collection VARIABLES. Let $X_{i}, X_{i+1}, \ldots, X_{j}(1 \leq i<j \leq n)$ be consecutive variables of the collection of variables VARIABLES such that the following conditions apply:

- All variables $X_{i}, \ldots, X_{j}$ take their value in the set of values VALUES,

- $i=1$ or $X_{i-1}$ does not take a value in VALUES,

- $j=n$ or $X_{j+1}$ does not take a value in VALUES.

We call such a set of variables a group. The constraint group_skip_isolated_item is true if all the following conditions hold:

- There are exactly NGROUP groups of variables,

- The number of variables of the smallest group is MIN_SIZE,

- The number of variables of the largest group is MAX_SIZE.

Inspired by group

?- group_skip_isolated_item $(2,2,1$

$[[\operatorname{var}-2],[\operatorname{var}-8],[\operatorname{var}-1],[\operatorname{var}-7],[\operatorname{var}-4]$,

$[\operatorname{var}-5],[\operatorname{var}-1],[\operatorname{var}-1],[\operatorname{var}-1]]$,

true.

$[[\operatorname{val}-0],[\operatorname{val}-2],[\operatorname{val}-4],[\operatorname{val}-6],[\operatorname{val}-8]])$.

: $0:$ not_in (VAR, VALUES)

\% 1 : in (VAR, VALUES)

group skip isolated item(MIN_SIZE, MAX_SIZE, NGROUP, VARIABLES, VALUES) :group_skip_isolated_item_max_size(MAX_SIZE, VARIABLES, VALUES), group_skip_isolated_item_min_size(MIN_SIZE, VARIABLES, VALUES), group skip isolated item_ngroup (NGROUP, VARIABLES, VALUES).

group_skip_isolated_item_max_size(MAX_SIZE, VARIABLES, VALUES) :- 
col_to_list (VALUES, LIST_VALUES),

list_to_fdset(LIST_VALUES, SET_OF_VALUES),

group_skip_isolated_item_signature (VARIABLES, SIGNATURE, SET_OF_VALUES),

automaton (SIGNATURE,

SIGNATURE, $0 \ldots 1$

$[\operatorname{source}(s), \operatorname{node}(i), \operatorname{sink}(t)]$

$[\operatorname{arc}(s, 0, s$

$\operatorname{arc}(s, s, t)$,

$\operatorname{arc}(i, 0, s,[\max (C, D), D])$,

$\operatorname{arc}(i, 1, i,[C \quad, D+1])$,

$\operatorname{arc}(i, \$, t,[\max (C, D), D])]$,

$[C, D],[0,0],[$ MAX_SIZE,_]).

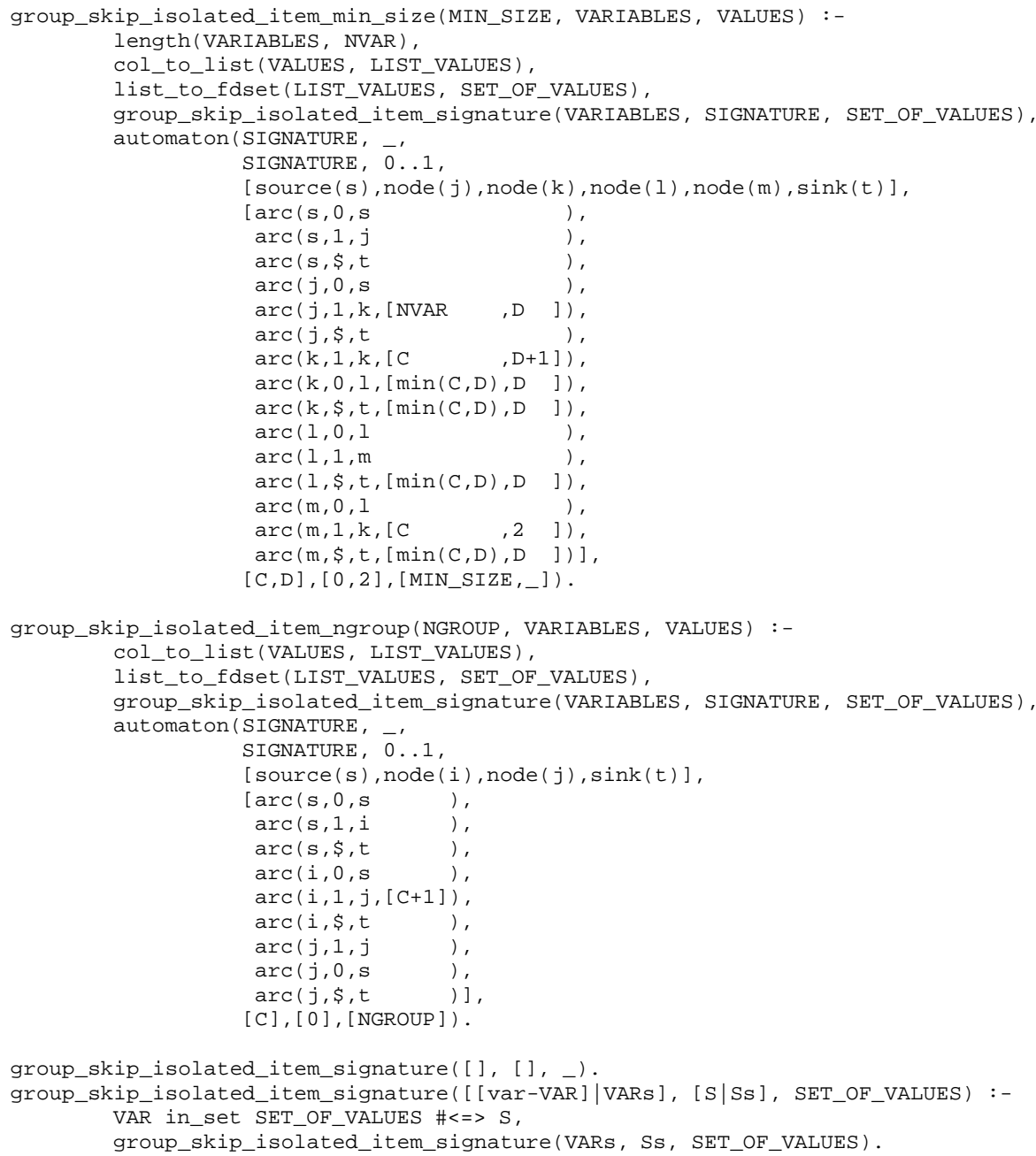

\section{A.21 in_(VAR, VALUES)}

Enforce the domain variable VAR to take a value within the values described by the VALUES collection. 


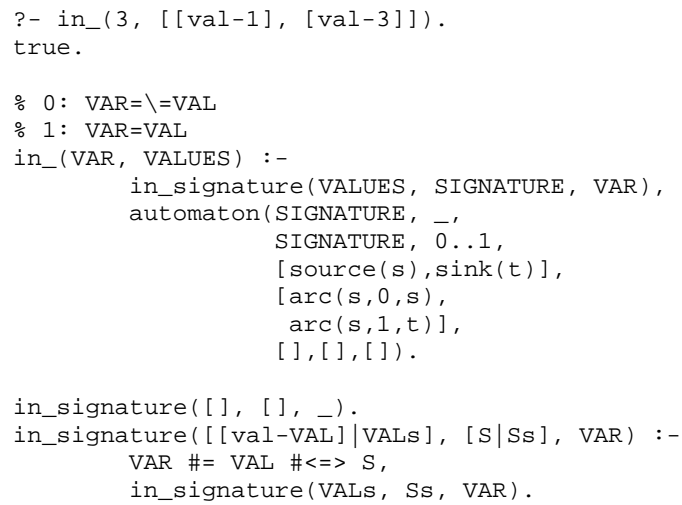

\section{A.22 in_same_partition (VAR1, VAR2, PARTITIONS)}

Enforce VAR1 and VAR2 to be respectively assigned to values $v_{1}$ and $v_{2}$ that both belong to a same partition of the collection PARTITIONS.

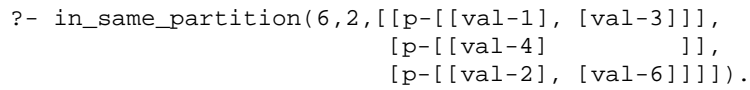

\section{A.23 inflexion (N, VARIABLES)}

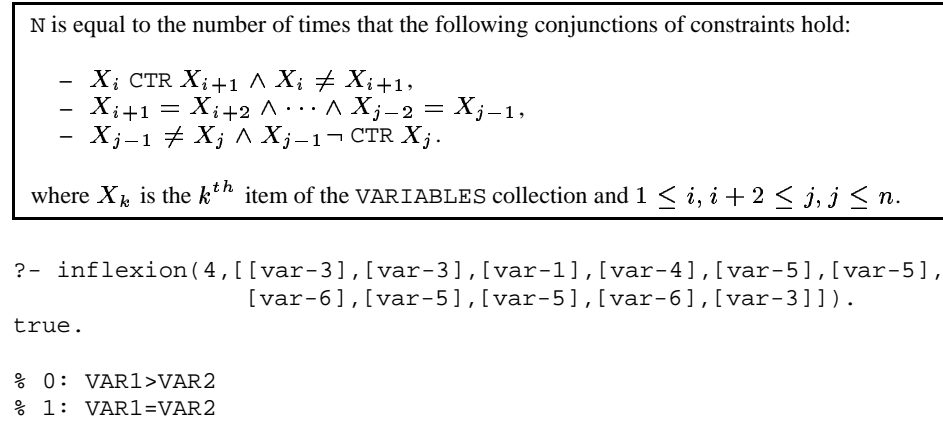




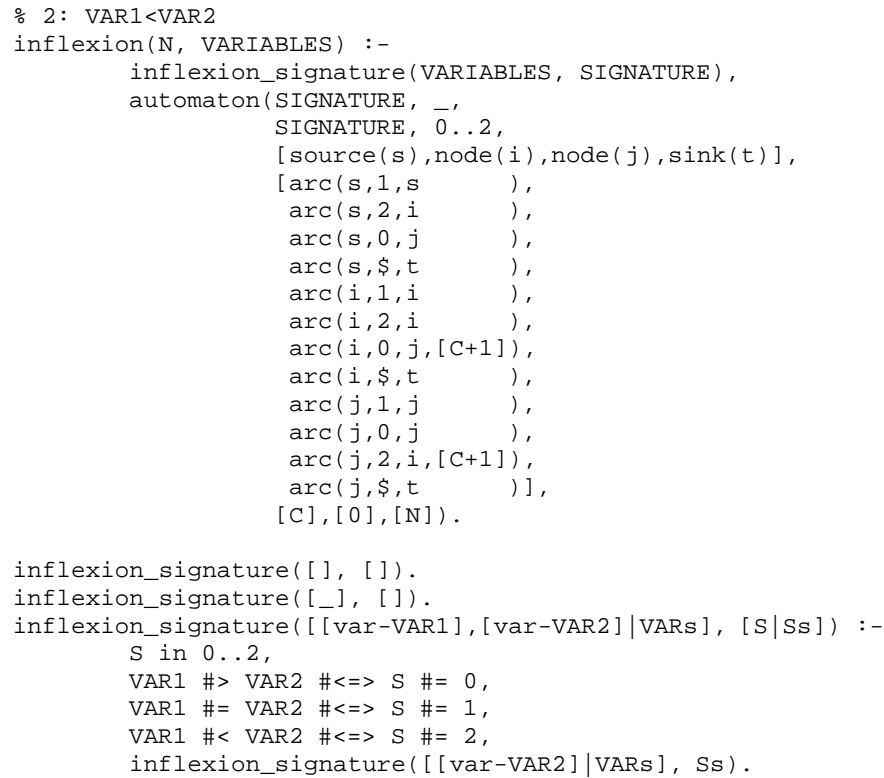

\section{A.24 lex_different (VECTOR1, VECTOR2)}

Vectors VECTOR1 and VECTOR2 differ from at least one component.

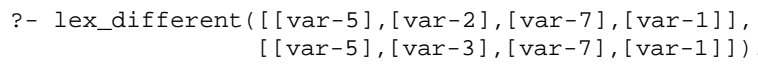

\section{A.25 lex_lesseq (VECTOR1, VECTOR2) [18]}

VECTOR1 is lexicographically less than or equal to VECTOR2.

Given two vectors, $\vec{X}$ and $\vec{Y}$ of $n$ components, $\left\langle X_{0}, \ldots, X_{n}\right\rangle$ and $\left\langle Y_{0}, \ldots, Y_{n}\right\rangle, \vec{X}$ is lexicographically less than or equal to $\vec{Y}$ iff $n=0$ or $X_{0}<Y_{0}$ or $X_{0}=Y_{0}$ and $\left\langle X_{1}, \ldots, X_{n}\right\rangle$ is lexicographically less than or equal to $\left\langle Y_{1}, \ldots, Y_{n}\right\rangle$.

?- lex_lesseq([[Var-5], [var-2], [var-3], [var-1]], $[[\operatorname{var}-5],[\operatorname{var}-2],[\operatorname{var}-6],[\operatorname{var}-2]])$. 
true.

\% 1: VAR1 < VAR2

$\div$ 2: VAR1 = VAR2

\% 3: VAR1 > VAR2

lex_lesseq(VECTOR1, VECTOR2) :-

lex lesseq signature(VECTOR1, VECTOR2, SIGNATURE), automaton (SIGNATURE,

SIGNATURE, 1 '. 3 ,

[ $\operatorname{source}(s), \operatorname{sink}(t)]$,

$[\operatorname{arc}(s, 1, t)$,

$\operatorname{arc}(s, 2, s)$,

$\operatorname{arc}(s, \$, t)]$

[]$,[],[])$.

lex_lesseq_signature ([], [], []).

lex_lesseq_signature([[Var-VAR1] |Xs], [[Var-VAR2]|Ys], [S|Ss]) :-

$S$ in $1 \ldots 3$,

VAR1 \#< VAR2 \#<=>S \#=1,

VAR1 \#= VAR2 \#<=>S \#=2,

VAR1 \# $>$ VAR2 \# $<\mathrm{S} \#=3$,

lex_lesseq_signature(Xs, Ys, Ss).

\section{A.26 longest_change (SIZE, VARIABLES, CTR)}

SIZE is the maximum number of consecutive variables of the collection VARIABLES for which constraint CTR holds in an uninterrupted way. We count a change when $X$ CTR $Y$ holds; $X$ and $Y$ are two consecutive variables of the collection VARIABLES. Derived from change.

?- longest_change (4, [ [var-8], [var-8], [var-3], [var-4], [var-1],

true. $[\operatorname{var}-1],[\operatorname{var}-5],[\operatorname{var}-5],[\operatorname{var}-2]],=\backslash=)$.

ㅇTR: =

0: VAR1 $=\backslash=$ VAR2

\% 1: VAR1=VAR2\$MMI\$ is the index of the variables corresponding to the maximum value of the collection of variables \$MVARIABLES\$.

\% CTR: $=\backslash=$

$0:$ VAR1 $=$ VAR2

1: $\operatorname{VAR} 1=\backslash=\operatorname{VAR} 2$

0: VAR1 $>=$ VAR2

1: VAR1 $<$ VAR2

CTR: $>=$

$0:$ VAR $1<$ VAR2

1: $\operatorname{VAR} 1>=$ VAR2

OCTR: >

0: VAR $1=<$ VAR2

1: VAR1 $>$ VAR2

CTR: $=<$

0: VAR1 $>$ VAR2

\% $1: \operatorname{VAR} 1=<\operatorname{VAR} 2$

longest_change(SIZE, VARIABLES, CTR) :-

longest_change_signature(VARIABLES, SIGNATURE, CTR), automaton (SIGNATURE,

SIGNATURE, $0 \ldots 1$

[ $\operatorname{source}(s), \operatorname{sink}(t)]$,

$[\operatorname{arc}(s, 1, s,[C, D+1])$,

$\operatorname{arc}(s, 0, s,[\max (C, D), 1])$,

$\operatorname{arc}(s, s, t,[\max (C, D), D])]$

$[C, D],[0,1],[S I Z E,-])$. 


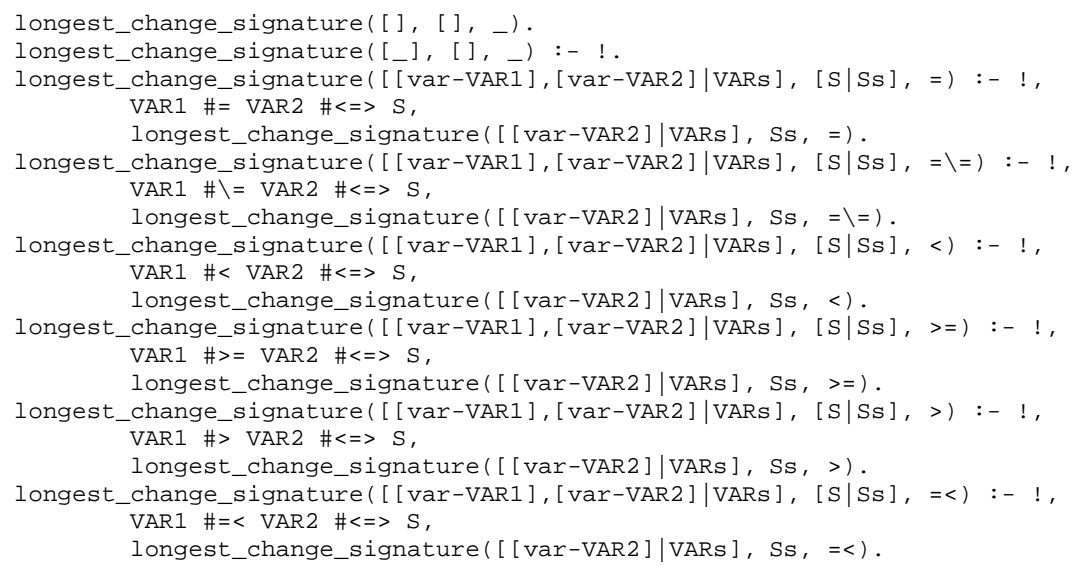

\section{A.27 max_index (MAX_INDEX, VARIABLES)}

MAX_INDEX is the index of the variables corresponding to the maximum value of the collection of variables VARIABLES.

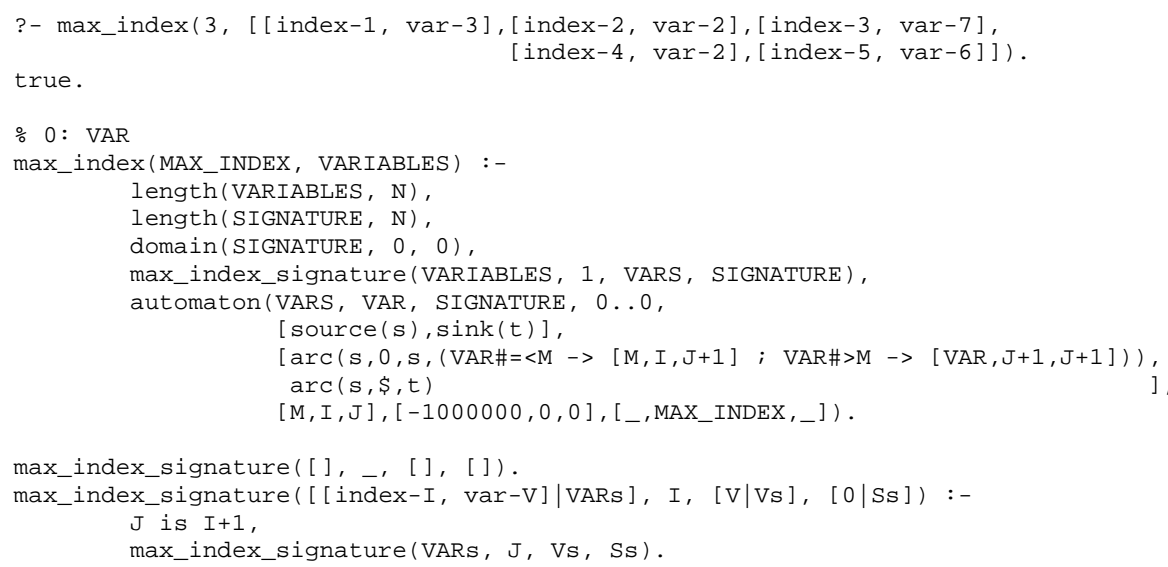

\section{A.28 maximum (MAX, VARIABLES)}

MAX is the maximum value of the collection of domain variables VARIABLES.

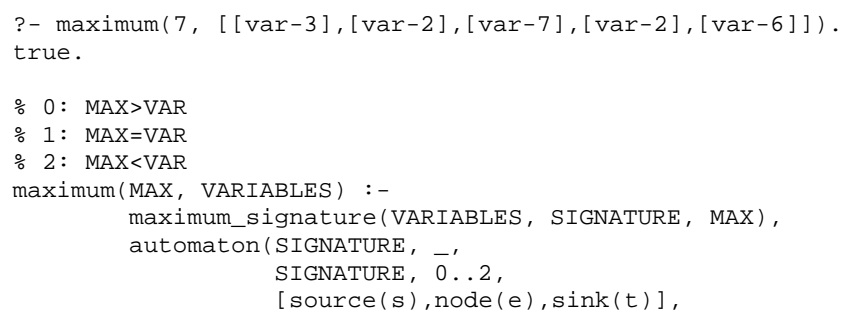




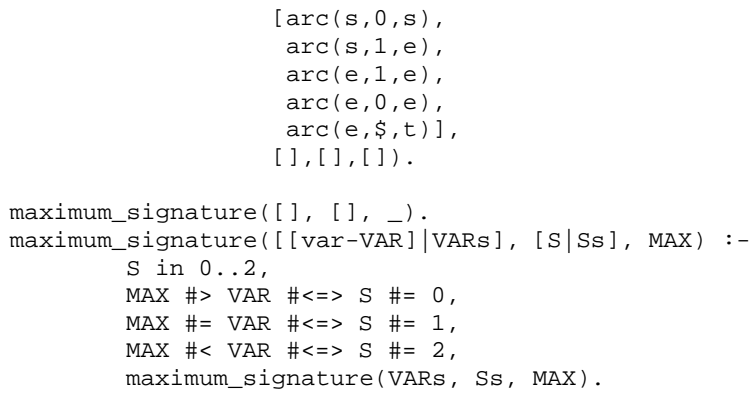

\section{A.29 not_all_equal (VARIABLES)}

The variables of the collection VARIABLES should take more than one single value.

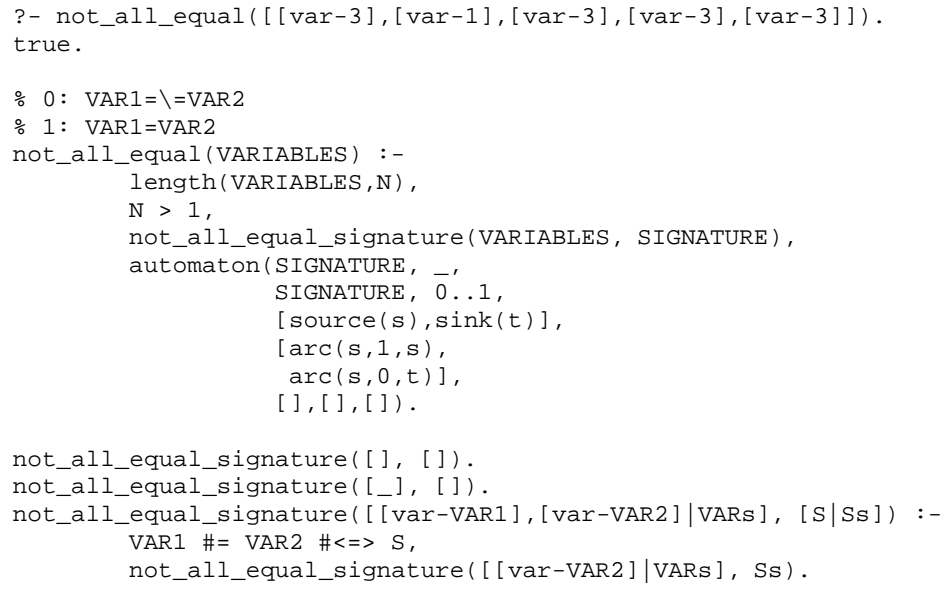

\section{A.30 not_in (VAR, VALUES)}

Remove the values of the VALUES collection from domain variable VAR.

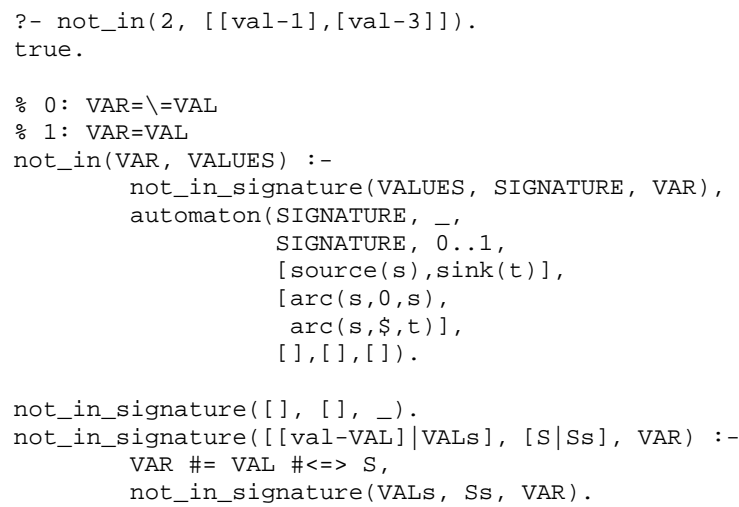




\section{A.31 pattern (VARIABLES) [11]}

We quote the definition from the original paper [11, page 157] introducing the pattern constraint.

We call a $k$-pattern any sequence of $k$ elements such that no two successive elements have the same value. Consider a

set $V=\left\{v_{1}, v_{2}, \ldots, v_{m}\right\}$ and a sequence $\mathbf{s}=\left\langle s_{1}, s_{2}, \ldots, s_{n}\right\rangle$ of elements of $V$. Consider now the sequence

$\left\langle v_{i_{1}}, v_{i_{2}}, \ldots, v_{i l}\right\rangle$ of the types of the successive stretches that appear in $\mathbf{s}$. Let $\mathcal{P}$ be a set of $k$-pattern. Vector $\mathbf{s}$ satisfies

$\mathcal{P}$ if and only if every subsequence of $k$ elements in $\left\langle v_{i_{1}}, v_{i_{2}}, \ldots, v_{i l}\right\rangle$ belongs to $\mathcal{P}$

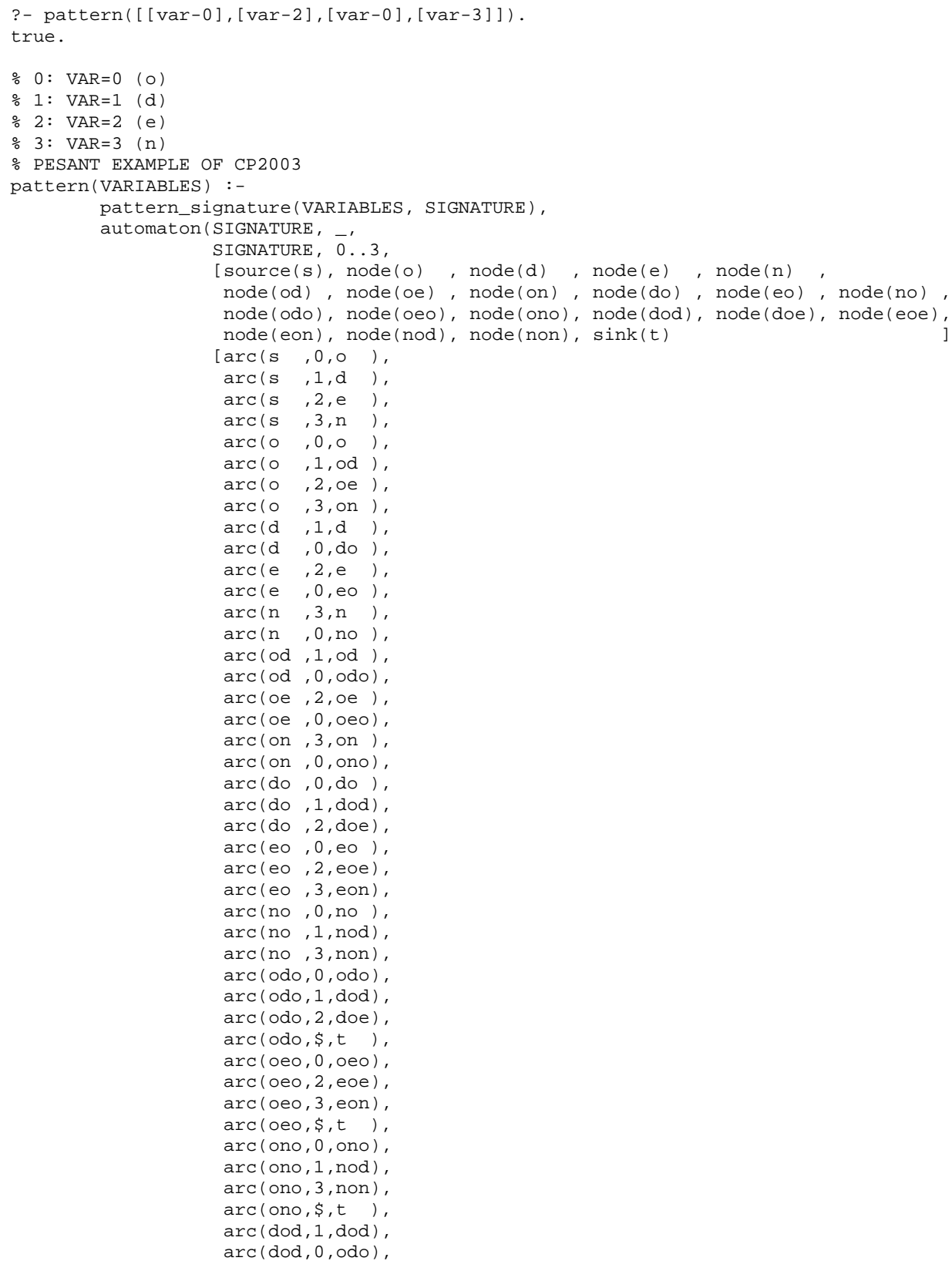




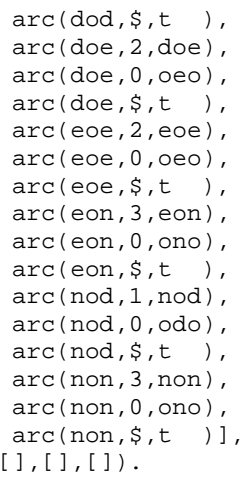

\section{A.32 peak (N, VARIABLES)}

A variable $V_{k}(1<k<m)$ of the sequence of variables VARIABLES $=V_{1}, \ldots, V_{m}$ is a peak if and only if there exist an $i(1<i \leq k)$ such that $V_{i-1}<V_{i}$ and $V_{i}=V_{i+1}=\ldots=V_{k}$ and $V_{k}>V_{k+1}$. N is the total number of peaks of the sequence of variables VARIABLES.

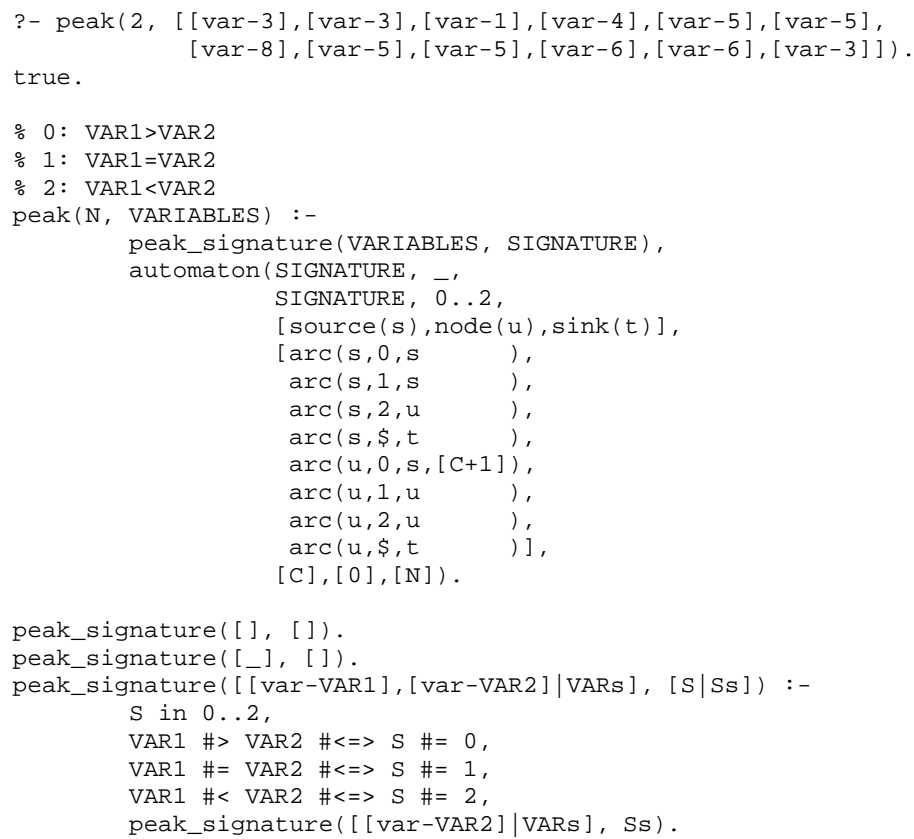

\section{A.33 sequence_folding (LETTERS)}

Express the fact that a sequence is folded in a way that no crossing occurs. A sequence is modelled by a collection of letters.

For each letter $l_{1}$ of a sequence, we indicate the next letter $l_{2}$ located after $l_{1}$ which is directly in contact with $l_{1}\left(l_{1}\right.$ itself if such a letter does not exist). Derived by Justin Pearson and motivated by RNA folding [15]. 


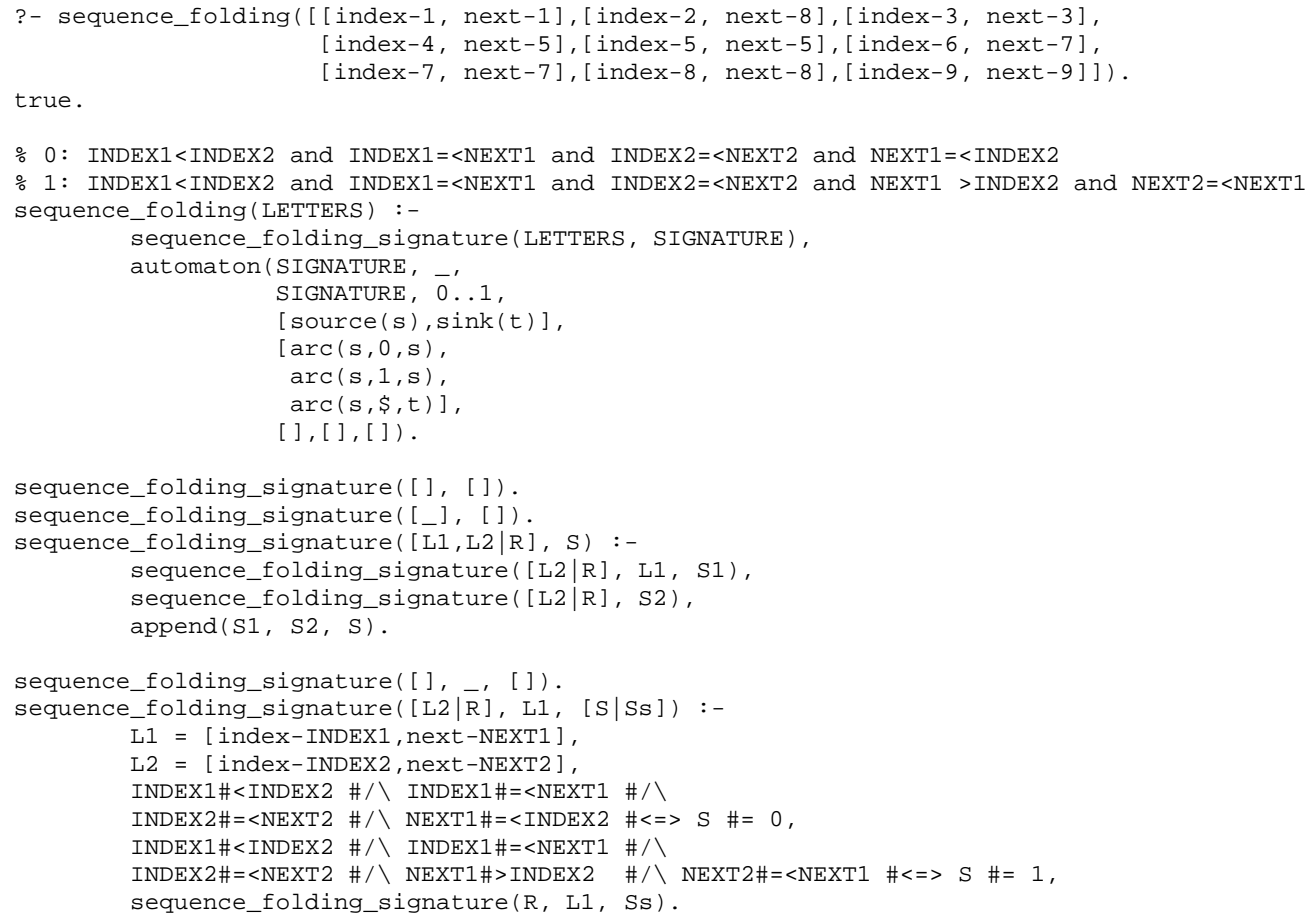

\section{A.34 sliding_card_skip0 (ATLEAST, ATMOST, VARIABLES, VALUES)}

Let $n$ be the total number of variables of the collection VARIABLES. A maximum non-zero set of consecutive variables $X_{i} . . X_{j}(1 \leq i \leq j \leq n)$ is defined in the following way:

- All variables $X_{i}, \ldots, X_{j}$ take a non-zero value,

- $i=1$ or $X_{i-1}$ is equal to 0

- $j=n$ or $X_{j+1}$ is equal to 0 .

Enforces that each maximum non-zero set of consecutive variables of the collection VARIABLES contains at least ATLEAST and at most ATMOST values from the collection of values VALUES.

?- sliding_card_skipo $(2,3$

$[[\operatorname{var}-0],[\operatorname{var}-7],[\operatorname{var}-2],[\operatorname{var}-9],[\operatorname{var}-0]$,

$[\operatorname{var}-0],[\operatorname{var}-9],[\operatorname{var}-4],[\operatorname{var}-9]]$

true.

[ [val-7], [val-9]]).

$\div 0: \operatorname{VAR}=0$

1: $\operatorname{VAR}=\backslash=0$ and not in (VAR, VALUES)

2: VAR $=\backslash=0$ and in (VAR, VALUES)

sliding_card_skipO (ATLEAST, ATMOST, VARIABLES, VALUES) :col_to_list (VALUES, LIST_VALUES)

list_to_fdset(LIST_VALUES, SET_OF_VALUES),

sliding_card_skip0_signature(VARIABLES, SIGNATURE, SET_OF_VALUES), automaton (SIGNATURE, -

SIGNATURE, $0 \ldots 2$

[ $\operatorname{source}(s), \operatorname{node}(i), \operatorname{sink}(t)]$,

$[\operatorname{arc}(s, 0, s$

$\operatorname{arc}(s, 1, i,[0]$

$\operatorname{arc}(s, 2, i,[1]$

$\operatorname{arc}(s, \$, t$

),
)'
), 


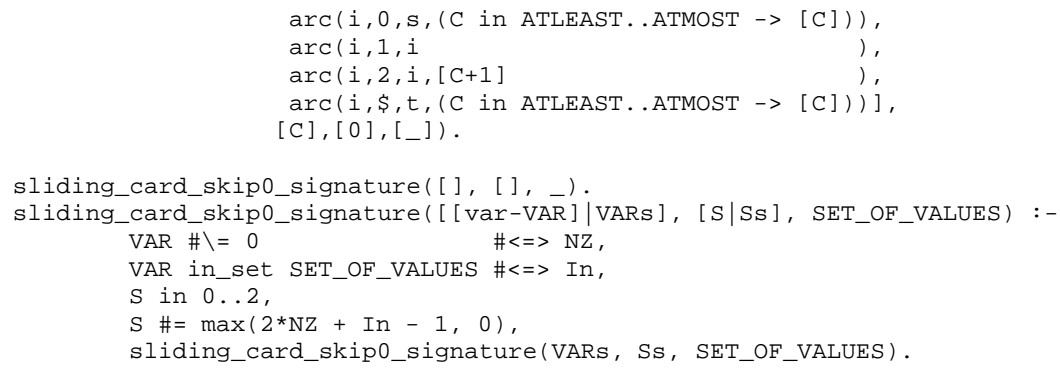

\section{A.35 smooth (NCHANGE, TOLERANCE, VARIABLES)}

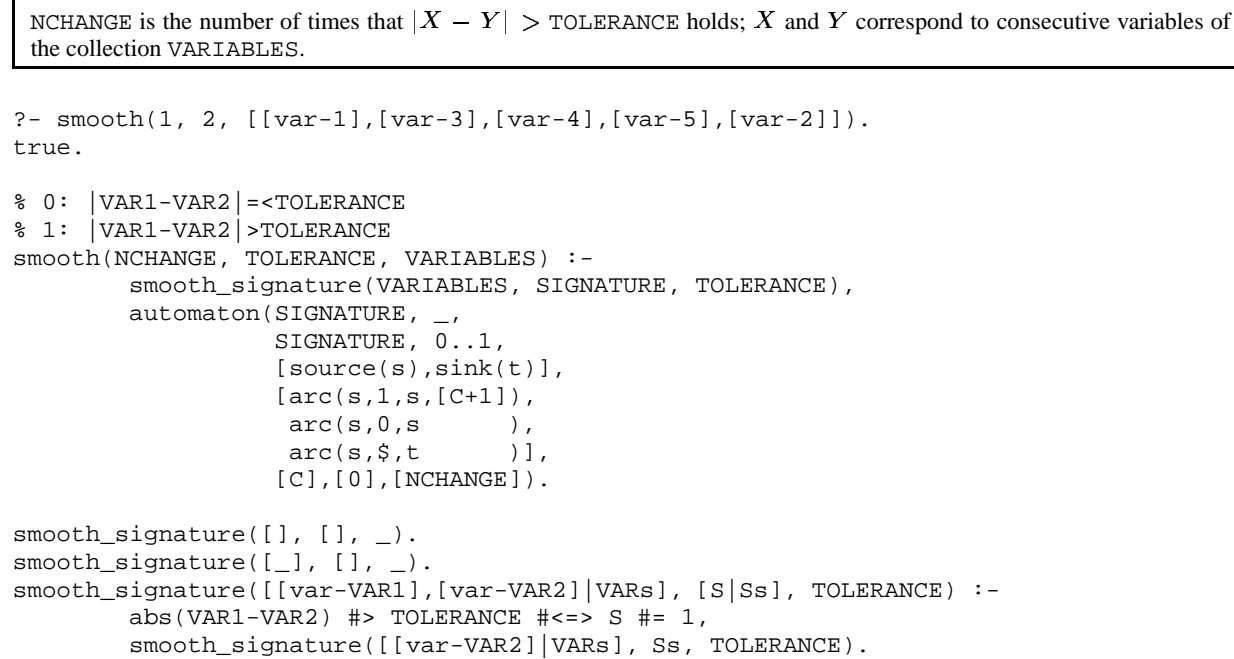

\section{A.36 top (N, VARIABLES) [8]}

A variable $V_{k}(1 \leq k \leq m)$ of the sequence of variables VARIABLES $=V_{1}, \ldots, V_{m}$ is a top if and only if there exist an $i(1 \leq i \leq k)$ such that $V_{i-1}<V_{i}$ and $V_{i}=V_{i+1}=\ldots=V_{k}$ and $V_{k}>V_{k+1}$ with convention that $V_{0}=V_{m+1}=0 . \bar{N}$ is the total number of tops of the sequence of variables VARIABLES.

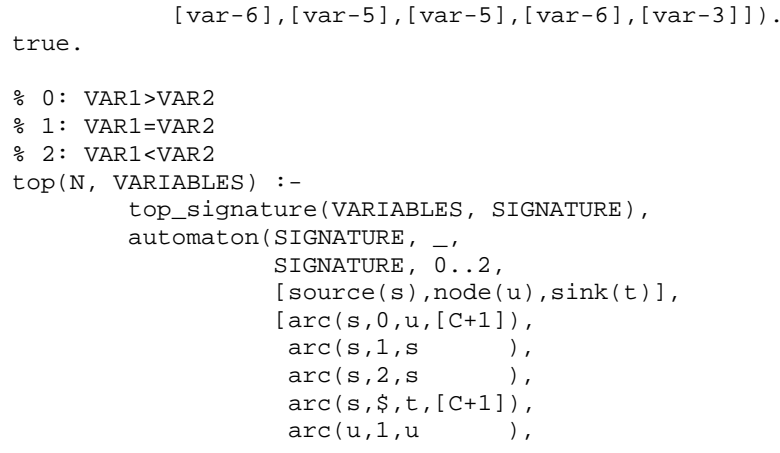




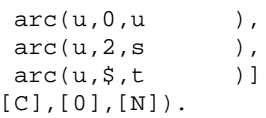

\section{A.37 two_quad_are_in_contact (QUADRANGLE1, QUADRANGLE2) [17]}

Enforce the following conditions on two quadrangles QUADRANGLE1 and QUADRANGLE2:

- For all dimensions $i$, except one dimension, the projections of QUADRANGLE1 and QUADRANGLE2 on $i$ have a nonempty intersection.

- For all dimensions $i$, the distance between the projections of QUADRANGLE1 and QUADRANGLE2 on $i$ is equal to 0 .

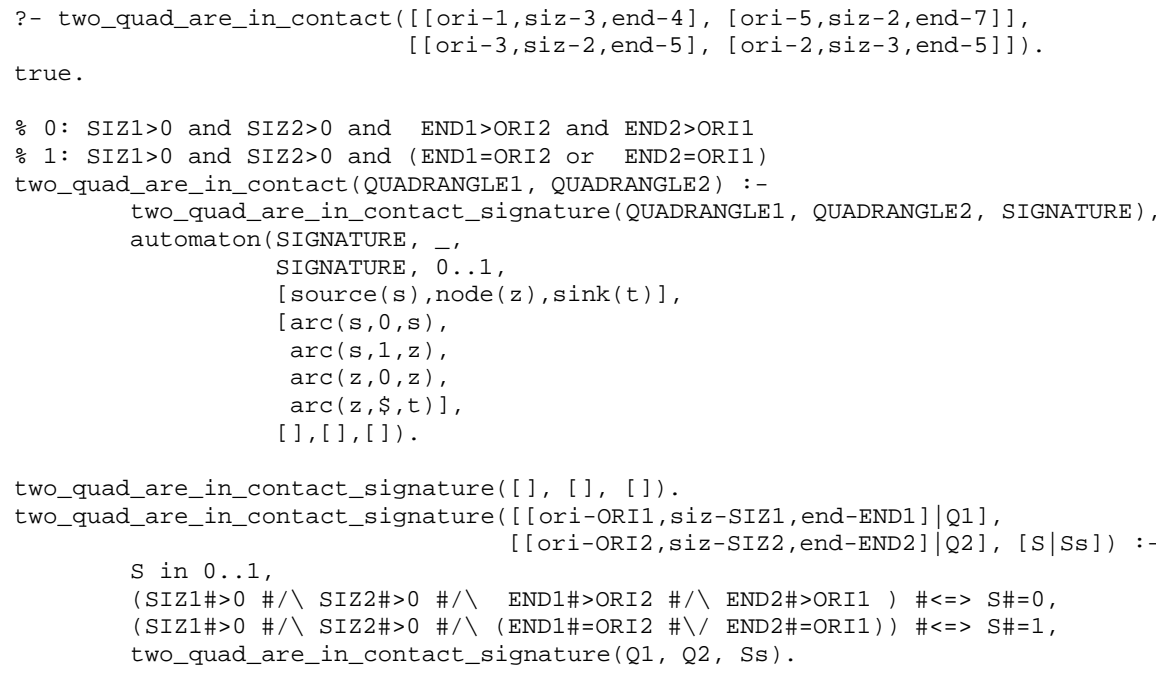

\section{A.38 two_quad_do_not_overlap (QUADRANGLE1, QUADRANGLE2) [17]}

For two quadrangles QUADRANGLE1 and QUADRANGLE2 enforce that there exist at least one dimension $i$ such that the projections on $i$ of QUADRANGLE 1 and QUADRANGLE2 do not overlap.

?- two_quad_do_not_overlap([[ori-2,siz-2,end-4], [ori-1,siz-3,end-4]],

true.

[ [ori-4, siz-4, end-8], [ori-3, siz-3, end-6]]).

응 $\mathrm{SIZ} 1=0$ or $\mathrm{SIZ} 2=0$ or $\mathrm{END} 1=<\mathrm{ORI} 2$ or $\mathrm{END} 2=<\mathrm{ORI} 1$

1: SIZ1>0 and SIZ2>0 and END1 >ORI2 and END2 >ORI1

two_quad_do_not_overlap (QUADRANGLE1, QUADRANGLE2) :-

two_quad_do_not_overlap_signature (QUADRANGLE1, QUADRANGLE2, SIGNATURE) automaton (SIGNATURE,

SIGNATURE, $0 \ldots 1$ 


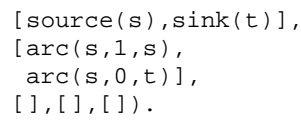

\section{A.39 valley (N, VARIABLES)}

A variable $V_{k}(1<k<m)$ of the sequence of variables VARIABLES $=V_{\mathbf{1}}, \ldots, V_{m}$ is a valley if and only if there exist an $i(1<i \leq k)$ such that $V_{i-1}>V_{i}$ and $V_{i}=V_{i+1}=\ldots=V_{k}$ and $V_{k}<V_{k+1}$. N is the total number of valleys of the sequence of variables VARIABLES.

?- $\operatorname{valley}(2,[[\operatorname{var}-3],[\operatorname{var}-3],[\operatorname{var}-1],[\operatorname{var}-4],[\operatorname{var}-5],[\operatorname{var}-5]$,

true.

$[\operatorname{var}-6],[\operatorname{var}-5],[\operatorname{var}-5],[\operatorname{var}-6],[\operatorname{var}-3]])$.

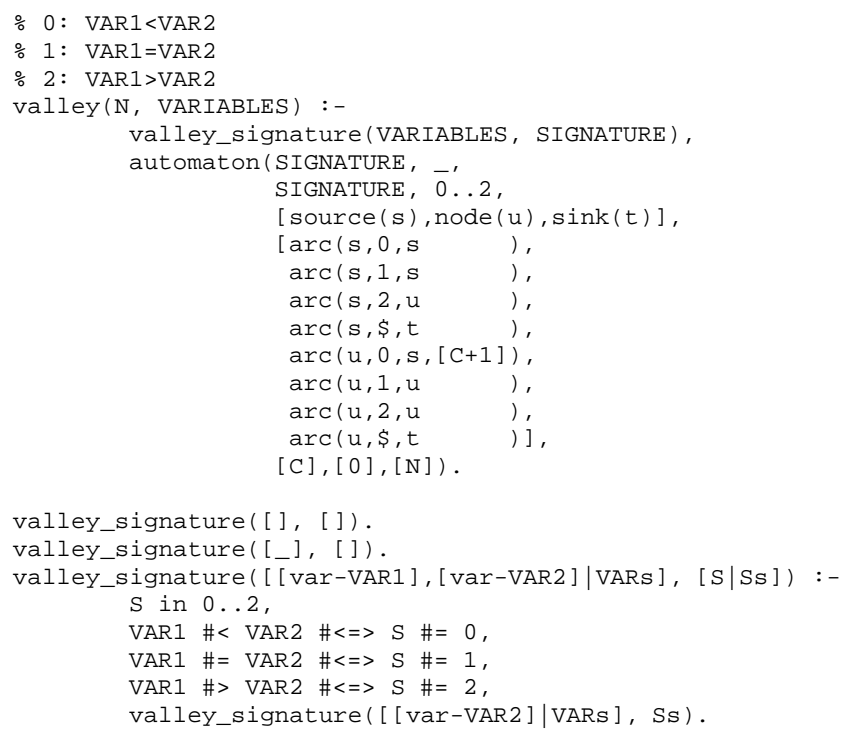

